\title{
Physics of Acoustic Radiation from Jet Engine Inlets
}

\author{
Christopher K. W. Tam ${ }^{1}$, Sarah A. Parrish ${ }^{2}$, Edmane Envia ${ }^{3}$ and Eugene W. Chien ${ }^{4}$ \\ ${ }^{1,2}$ Florida State University, Tallahassee, FL 32306-4510 \\ ${ }^{3}$ NASA Glenn Research Center, Cleveland, OH 44135 \\ ${ }^{4}$ Goodrich Aerostructures Group, Chula Vista, CA 91910
}

\begin{abstract}
Numerical simulations of acoustic radiation from a jet engine inlet are performed using advanced computational aeroacoustics (CAA) algorithms and high-quality numerical boundary treatments. As a model of modern commercial jet engine inlets, the inlet geometry of the NASA Source Diagnostic Test (SDT) is used. Fan noise consists of tones and broadband sound. This investigation considers the radiation of tones associated with upstream propagating duct modes. The primary objective is to identify the dominant physical processes that determine the directivity of the radiated sound. Two such processes have been identified. They are acoustic diffraction and refraction. Diffraction is the natural tendency for an acoustic wave to follow a curved solid surface as it propagates. Refraction is the turning of the direction of propagation of sound waves by mean flow gradients. Parametric studies on the changes in the directivity of radiated sound due to variations in forward flight Mach number and duct mode frequency, azimuthal mode number, and radial mode number are carried out. It is found there is a significant difference in directivity for the radiation of the same duct mode from an engine inlet when operating in static condition and in forward flight. It will be shown that the large change in directivity is the result of the combined effects of diffraction and refraction.
\end{abstract}

\section{Introduction}

Acoustic radiation from jet engine inlets has been studied experimentally ${ }^{1-9}$, analytically ${ }^{10-13}$ and computationally ${ }^{14-26}$ by a number of investigators in the past. These studies provide a variety of useful and interesting information. They also provide prediction capabilities and methods. It is known that sound radiated out of an engine inlet consists of both broadband noise and tones. Broadband fan noise is random and chaotic and is best studied statistically. Tones, on the other hand, which are generated by the fan rotating at high speeds and by the cutting of the rotor wake by the stator blades, are highly organized and propagate coherently. In the inlet duct, tones propagate as duct modes. Because duct modes are coherent propagating entities, they are readily open to analysis and numerical simulation. Their propagating characteristics are also easy to understand. Duct mode propagation from the fan face to the far field is the subject of the present investigation.

It is our belief that the mechanisms that influence the radiation of duct modes operate independent of the engine inlet geometry; the geometry of the engine inlet does, however, alter the relative importance of the various mechanisms. For this reason, this study uses primarily the inlet geometry of the NASA Source Diagnostic Test (SDT) fan, which has internal fan duct diameter of approximately 22 inches at the fan face. The fan has 22 blades. The inlet geometry of the SDT fan is typical of most modern jet engines.

There is significant complexity in the duct mode radiation processes. To illustrate this point, consider the radiation patterns associated with the NASA SDT fan inlet in Figs. 1 and 2. The flow Mach number at the fan face is the same for both cases: $M_{\mathrm{fan}}=0.4$. The duct mode radiating out of the inlet is also the same in each case and is defined with azimuthal mode number $m=22$, radial mode number $n=1$, and frequency $f=6400 \mathrm{~Hz}$. Fig. 1 shows

${ }^{1}$ Robert O. Lawton Distinguished Professor, Department of Mathematics, AIAA Fellow.

2 Research Associate, Department of Mathematics.

${ }^{3}$ Research Aerospace Engineer, Acoustics Branch, AIAA Associate Fellow.

${ }^{4}$ Staff Engineer, Acoustics 
the pressure contour pattern for the engine at static condition, i.e., no forward flight. Fig. 2 shows the pressure contour pattern when the engine is moving with forward flight Mach number 0.2. It is obvious that the dominant directions of radiation in the two cases are substantially different. But why are they so different for such a low flight Mach number? Currently, a valid explanation does not appear to be available in the open literature.

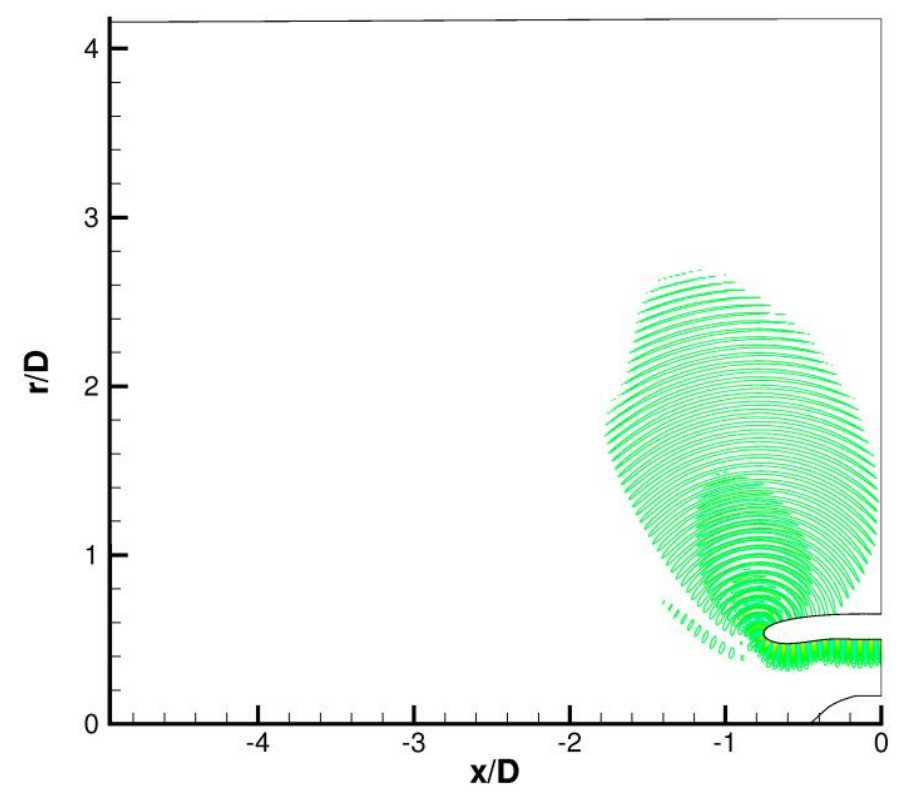

Figure 1. Computed pressure contour pattern of an $m=22, n=1$ duct mode at $6400 \mathrm{~Hz}$ radiating out of the SDT fan inlet. $M_{\text {fan }}=0.4, M_{\text {flight }}=0.0$ (static condition).

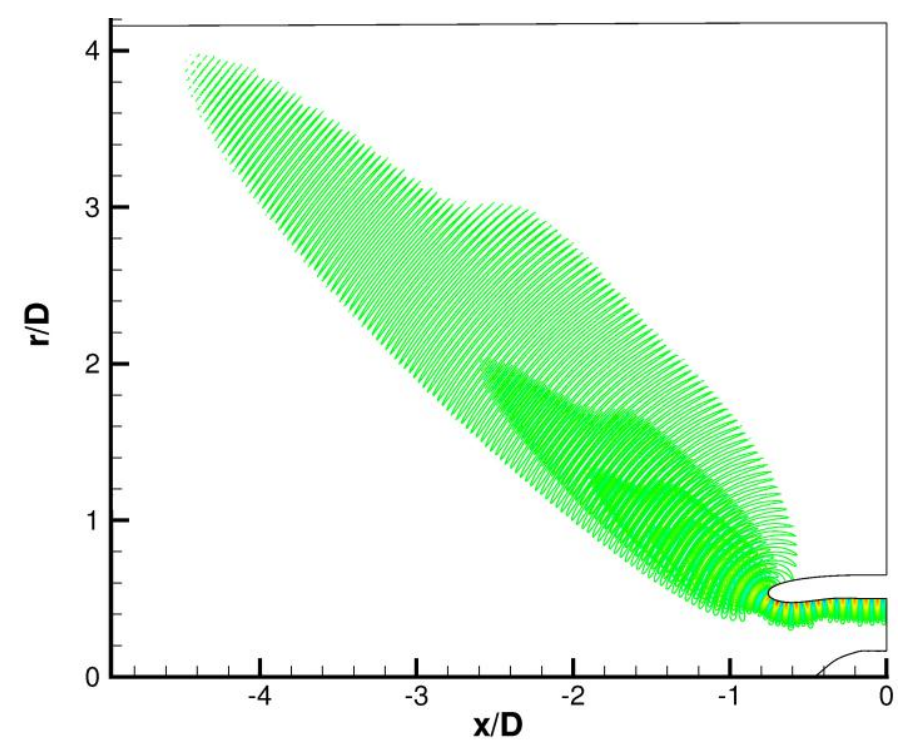

Figure 2. Computed pressure contour pattern of an $m=22, n=1$ duct mode at $6400 \mathrm{~Hz}$ radiating out of the SDT fan inlet. $M_{\text {fan }}=0.4, M_{\text {flight }}=0.2$.

Thus, in spite of past studies, there seems to be a need for further effort in the investigation of the flow physics that controls and influences the acoustic radiation processes. This is the purpose of the present investigation. To accomplish this goal, numerical simulation using advanced computational aeroacoustics (CAA) methods is used. We would like to point out that, for the stated purpose, numerical simulation has some significant advantages over an experimental investigation. This does not mean that experiments are unimportant. Actually, they are absolutely necessary for validation and other purposes. A few of these advantages are listed below. 
1. In a numerical simulation, there is full access to space and time data inside and outside an engine inlet. They include both the mean flow and acoustic disturbances. These types of data are needed in order to understand the physical processes involved in the radiation of a duct mode. In principle, these quantities can also be measured experimentally. But to do so would require an extraordinary effort; therefore, it has never been done.

2. Computationally, the amount of effort required to simulate engine inlet radiation with and without forward flight is about the same. Experimentally, studying forward flight effect in a laboratory would require the use of a wind tunnel, which introduces background wind tunnel noise and possible extraneous tones from the facility. Complications also arise from wall reflection, if a closed wind tunnel is used, and from shear layer correction, if an open wind tunnel is used.

3. Numerical simulation allows an easy study of the radiation of an individual duct mode. A parametric study of the variation of mode frequency and azimuthal and radial mode numbers can easily be carried out. Experimentally, generating a single duct mode is no trivial matter. Usually, the measured pressure signal contains a multitude of modes. Unscrambling the measured pressure data in order to recover individual mode information is not an easy task.

In this paper, it is our intent to show that duct mode radiation from a jet engine inlet is greatly influenced by the effects of diffraction and refraction. Here, diffraction refers to the inherent characteristic of a duct mode to follow and remain attached to the wall of the engine casing as it propagates and eventually radiates away. Refraction is the turning of the direction of propagation of a duct mode by mean flow gradients. The degree of influence of these two effects is, however, highly dependent on fan face Mach number, $M_{\text {fan }}$, forward flight Mach number, $M_{\text {flight }}$, azimuthal and radial mode numbers, $(m, n)$, and frequency, $f$. The sum of all the individual effects ultimately determines the dominant direction of radiation for a duct mode. The present investigation focuses on finding the dependence of the diffraction and refraction effects on each of the above parameters. Reporting and explaining these results forms the main body of this paper. In addition, we will document the CAA methods and algorithms used, as they may be of interest to other investigators. To assure that our simulations are accurate, we have performed validation tests by comparing the computed directivity with experimental measurements.

The rest of the paper is as follows: Section 2 discusses the computational grid, computational model and algorithm, as well as various types of boundary conditions used in the present numerical simulations. Section 3 reports the results of a series of computer code validation tests. Directivity data from a JT15D engine experiment ${ }^{1,2}$ is used for comparison. Section 4 presents computed mean flow data, both with and without forward flight, for the NASA SDT engine inlet. Acoustic results are reported in Section 5. They include a detailed study of the effects of diffraction and refraction. An explanation for the large impact of forward flight on the direction of duct mode radiation is presented. A parametric study showing the effects of forward flight Mach number, duct mode frequency, and azimuthal and radial mode numbers on the radiation pressure contour pattern and directivity has been carried out. The results of this parametric study are reported in Section 6. The impact of diffraction and refraction effects on these results is explained. Section 7 gives a summary and conclusion of this work.

\section{Computational Model, Grid Design, and Computational Algorithm}

In this section, information on the computational domain, computational model, grid design and computational algorithm used in the present numerical simulation of jet engine inlet radiation is provided.

\subsection{Grid design}

Fig. 3 shows the computational domain in physical space. The engine inlet is at the lower-right corner. The internal diameter, $D$, of the casing at the fan face is used as the length scale. For the NASA STD fan, $D$ is approximately 22 inches. There are twenty-two fan blades. The computational domain extends five diameters $(5 D)$ in front of the fan face and four diameters $(4 D)$ in the sideline direction. This is the size of the domain used for near field pressure contour pattern computation. For validation directivity computations, the domain is extended further to the left by another $5 D$. To generate a body-fitted grid, a conformal mapping method is used ${ }^{27}$. The engine casing and hub is first extended by symmetry about the vertical line passing through the fan face as shown in Fig. 4. 


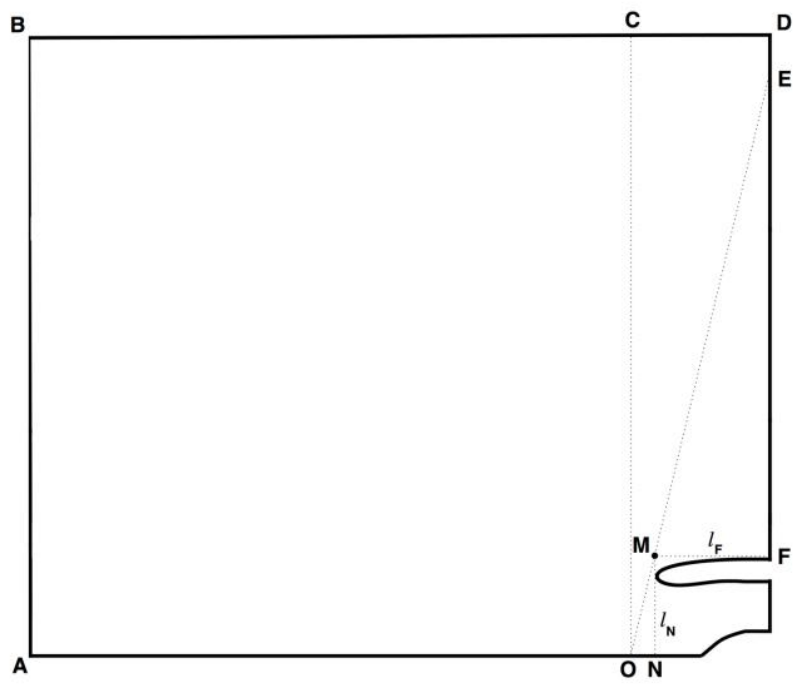

Figure 3. Computational domain for near field pressure contour pattern computations.

Doublets are placed along the centerlines of the three bodies in Fig. 4. A large set of enforcement points on the surface of the bodies is selected. The strengths of the doublets are then chosen so the enforcement points are mapped onto three parallel slits in the computational plane as shown in Fig. 5. Because the number of enforcement points is much higher than the number of doublets, the number of linear algebraic equations derived from mapping to the slits condition is far higher than the number of unknowns. It is an over-determined system. Such a system can, however, be solved by a mean-least-squared method known as the method of normal matrix. Details of the conformal mapping method are provided in Ref. [27].

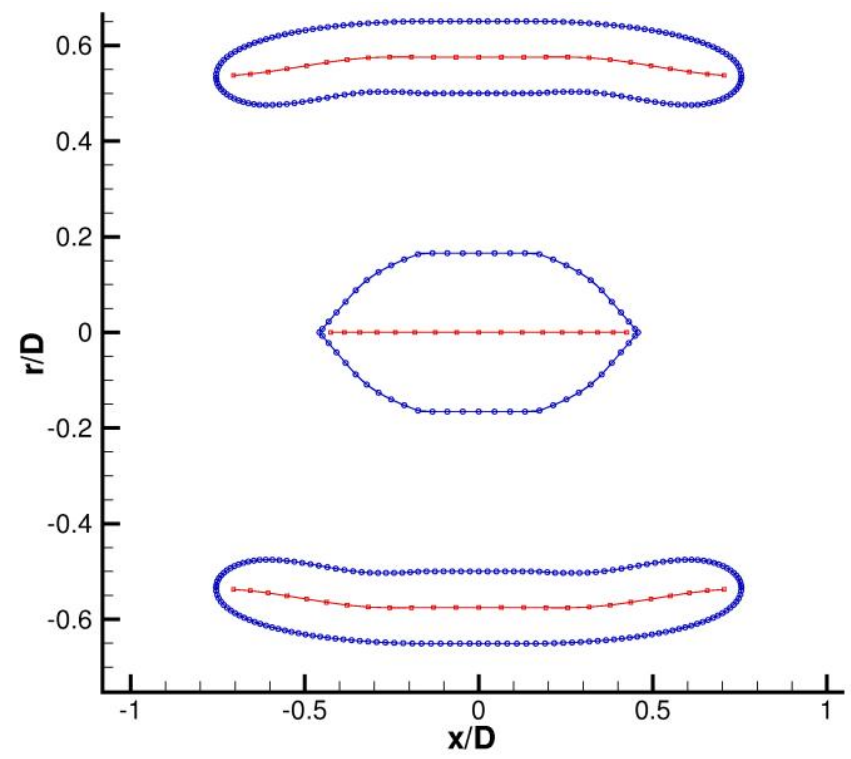

Figure 4. Locations of doublets and enforcement points for conformal mapping of the engine inlet into 3 slits. 


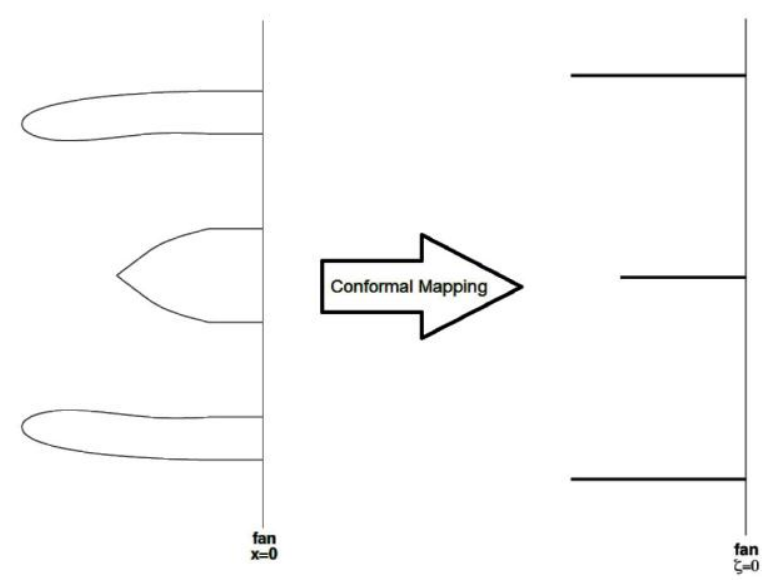

Figure 5. Mapping of engine inlet into three slits in the computational domain.

In the mapped plane, i.e., the computation plane or the $\varsigma-\eta$ plane, the inlet consists of three slits. A Cartesian grid can easily be fitted into the three-slits geometry in the computational domain, as shown in Fig. 6. Because of axisymmetry, only the top half of the inlet needs be considered. A multiple-size grid is used to ensure accuracy and efficiency. The finest grid is used around the walls of the casing and the hub, as shown in Fig. 6. In moving outward from these finest regions, three grid-size changes occur, each increasing the grid size by a factor of two. The result is a total of four grid sizes, where the grid size of the coarsest region is eight times that of the finest region. This grid arrangement is adopted in anticipation of using the multi-size-mesh and multi-time-step DRP scheme $^{28}$ as the computational algorithm.

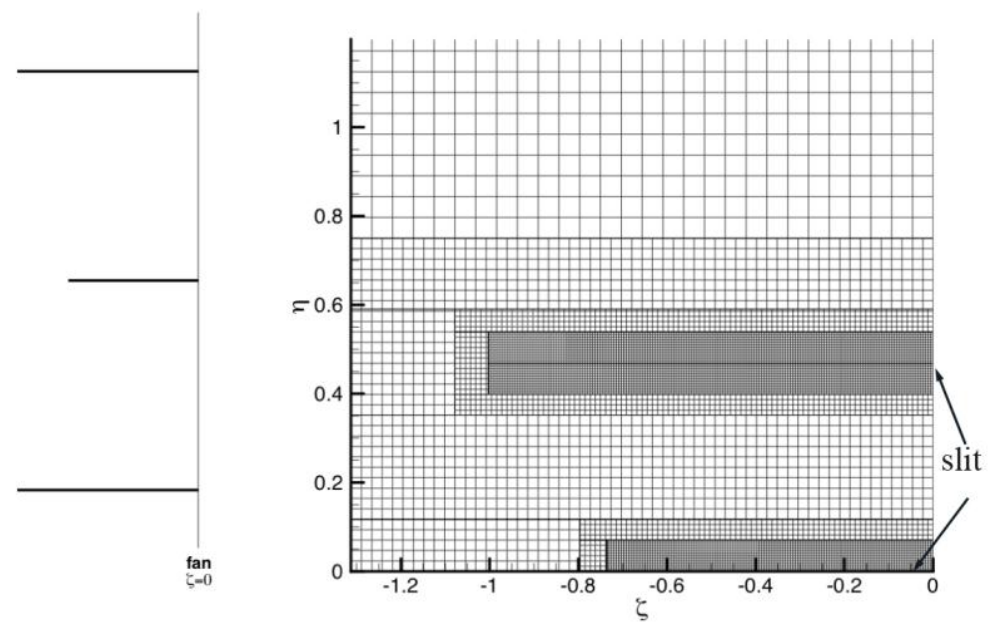

Figure 6. Multi-size Cartesian grid in the computational plane or the $\varsigma-\eta$ plane.

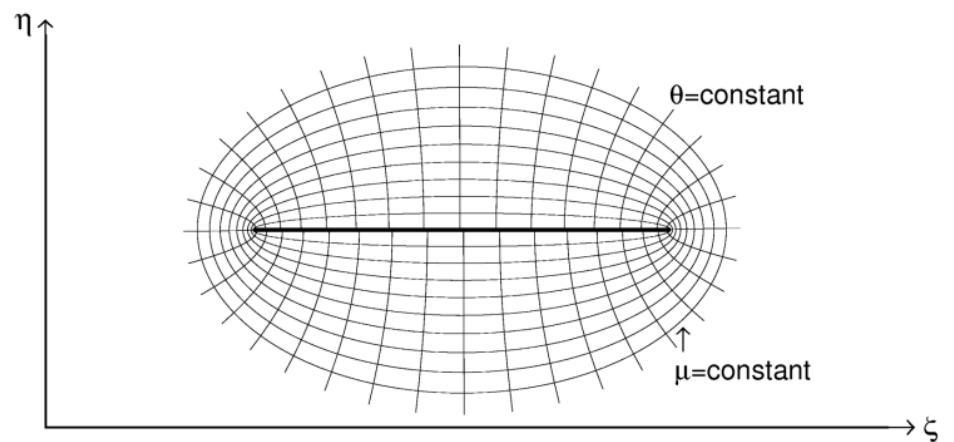

Figure 7. Elliptic coordinate lines form a body-fitted grid around a slit. 
In the computational domain, both the casing and the hub are mapped into slits. A Cartesian grid would provide adequate resolution everywhere except at the tip of the slit. To improve resolution near the tips of the slits, an elliptic grid ( $\mu-\theta$ coordinates), which forms a body-fitted grid to a slit, is introduced (see Fig. 7). A lensshaped portion of the elliptic grid, as shown in Fig. 8, is included in the computation. The Cartesian grid computation ends at the boundary of the rectangular box shown in Fig. 8. The computation in the elliptic grid is confined to the lens-shaped region in this figure. In the overlapping region, data are transferred by interpolation from one grid to the other, typical of the overset grid method ${ }^{29}$. In the physical plane, the grid design around the casing and the hub is shown in Fig. 9. The computational grid in the physical plane is displayed in Fig. 10. Note in Figs. 9 and 10 , the grids have been coarsened for illustrative purposes.

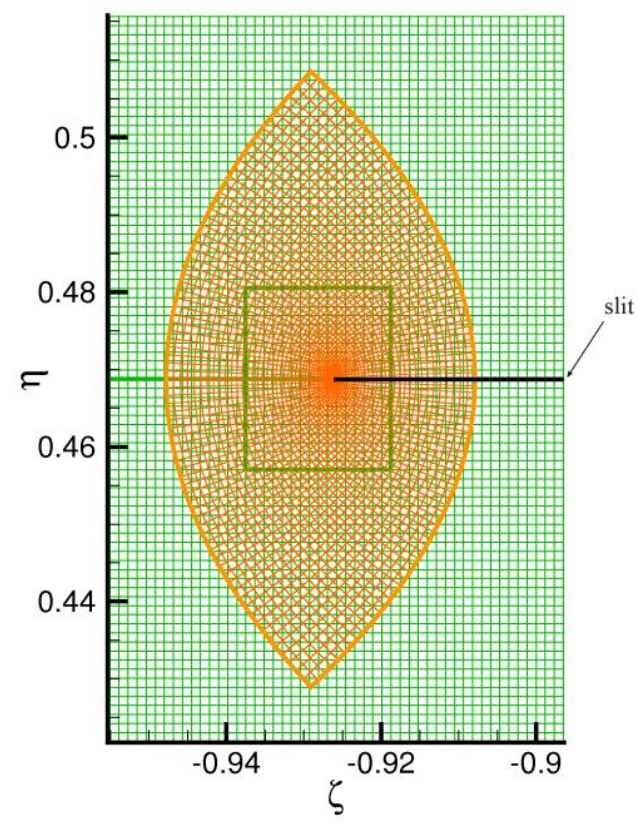

Figure 8. A lens-shaped region of the elliptic grid of Fig. 7 is used to improve resolution at the tip of a slit.
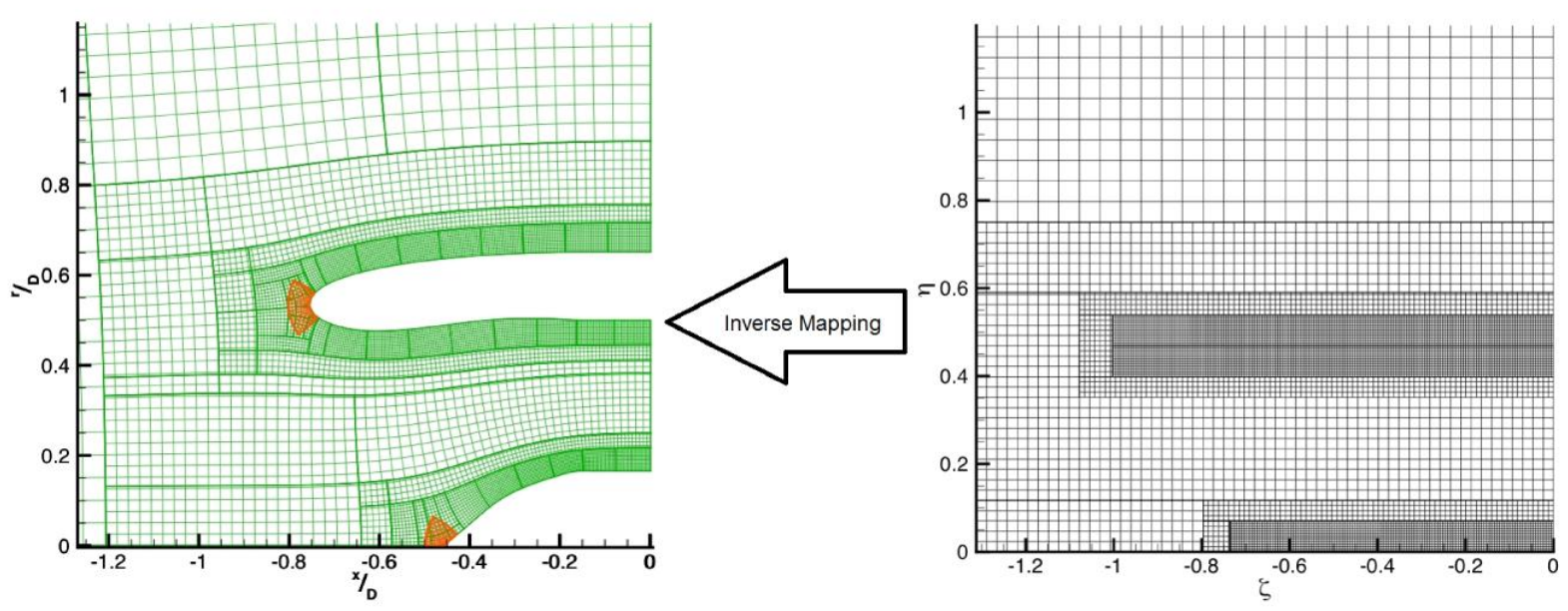

Figure 9. Enlarged grid design in the computational and the physical planes. 


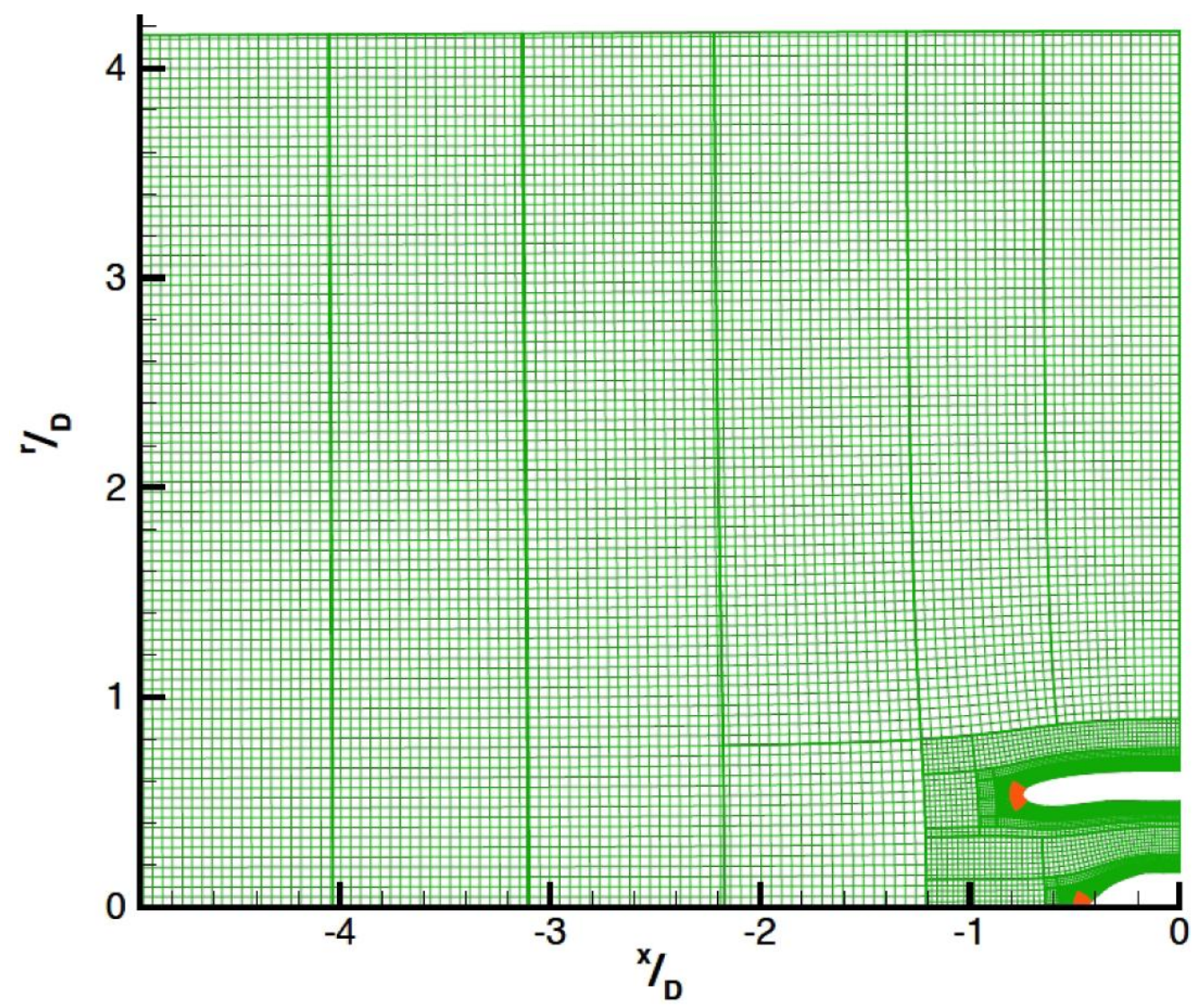

Figure 10. The computational grid in the physical $x$ - $r$ plane. Engine inlet is at the lower-right corner.

\subsection{Mean flow computation}

For our computation, the gas is assumed to be inviscid. The mean flow is axisymmetric, so there is no azimuthal dependence. The governing equations are the Euler equations, which written in the computational coordinates $(\varsigma, \eta)$ in dimensionless form are:

$$
\begin{gathered}
\text { length scale }=D \text { (internal diameter of duct at fan face) } \\
\text { velocity scale }=a_{0} \text { (ambient sound speed) } \\
\text { time scale }=D / a_{0} \\
\text { density scale }=\rho_{0} \text { (ambient gas density) } \\
\text { pressure scale }=\rho_{0} a_{0}^{2} \\
\frac{\partial \rho}{\partial t}+v\left(\frac{\partial \rho}{\partial \varsigma} \frac{\partial \varsigma}{\partial r}+\frac{\partial \rho}{\partial \eta} \frac{\partial \eta}{\partial r}\right)+u\left(\frac{\partial \rho}{\partial \varsigma} \frac{\partial \varsigma}{\partial x}+\frac{\partial \rho}{\partial \eta} \frac{\partial \eta}{\partial x}\right)+\rho\left(\frac{\partial v}{\partial \varsigma} \frac{\partial \varsigma}{\partial r}+\frac{\partial v}{\partial \eta} \frac{\partial \eta}{\partial r}+\frac{v}{r}+\frac{\partial u}{\partial \varsigma} \frac{\partial \varsigma}{\partial x}+\frac{\partial u}{\partial \eta} \frac{\partial \eta}{\partial x}\right)=0 \\
\frac{\partial u}{\partial t}+v\left(\frac{\partial u}{\partial \varsigma} \frac{\partial \varsigma}{\partial r}+\frac{\partial u}{\partial \eta} \frac{\partial \eta}{\partial r}\right)+u\left(\frac{\partial u}{\partial \varsigma} \frac{\partial \varsigma}{\partial x}+\frac{\partial u}{\partial \eta} \frac{\partial \eta}{\partial x}\right)+\frac{1}{\rho}\left(\frac{\partial p}{\partial \varsigma} \frac{\partial \varsigma}{\partial x}+\frac{\partial p}{\partial \eta} \frac{\partial \eta}{\partial x}\right)=0 \\
\frac{\partial v}{\partial t}+v\left(\frac{\partial v}{\partial \varsigma} \frac{\partial \varsigma}{\partial r}+\frac{\partial v}{\partial \eta} \frac{\partial \eta}{\partial r}\right)+u\left(\frac{\partial v}{\partial \varsigma} \frac{\partial \varsigma}{\partial x}+\frac{\partial v}{\partial \eta} \frac{\partial \eta}{\partial x}\right)+\frac{1}{\rho}\left(\frac{\partial p}{\partial \varsigma} \frac{\partial \varsigma}{\partial r}+\frac{\partial p}{\partial \eta} \frac{\partial \eta}{\partial r}\right)=0 \\
\frac{\partial p}{\partial t}+v\left(\frac{\partial p}{\partial \varsigma} \frac{\partial \varsigma}{\partial r}+\frac{\partial p}{\partial \eta} \frac{\partial \eta}{\partial r}\right)+u\left(\frac{\partial p}{\partial \varsigma} \frac{\partial \varsigma}{\partial x}+\frac{\partial p}{\partial \eta} \frac{\partial \eta}{\partial x}\right)+\gamma p\left(\frac{\partial v}{\partial \varsigma} \frac{\partial \varsigma}{\partial r}+\frac{\partial v}{\partial \eta} \frac{\partial \eta}{\partial r}+\frac{v}{r}+\frac{\partial u}{\partial \varsigma} \frac{\partial \varsigma}{\partial x}+\frac{\partial u}{\partial \eta} \frac{\partial \eta}{\partial x}\right)=0
\end{gathered}
$$


where $\varsigma(x, r), \eta(x, r)$ and inverses $x(\varsigma, \eta), r(\varsigma, \eta)$ are given by the conformal mapping function. The variables $(u, v)$ are the velocity components in the axial and radial directions, respectively.

For computation in the overset elliptic grid, a further change of variables from $(\varsigma, \eta)$ to $(\mu, \theta)$ is necessary. This step is fairly straightforward, so the governing equations in $(\mu, \theta)$ coordinates will not be written out.

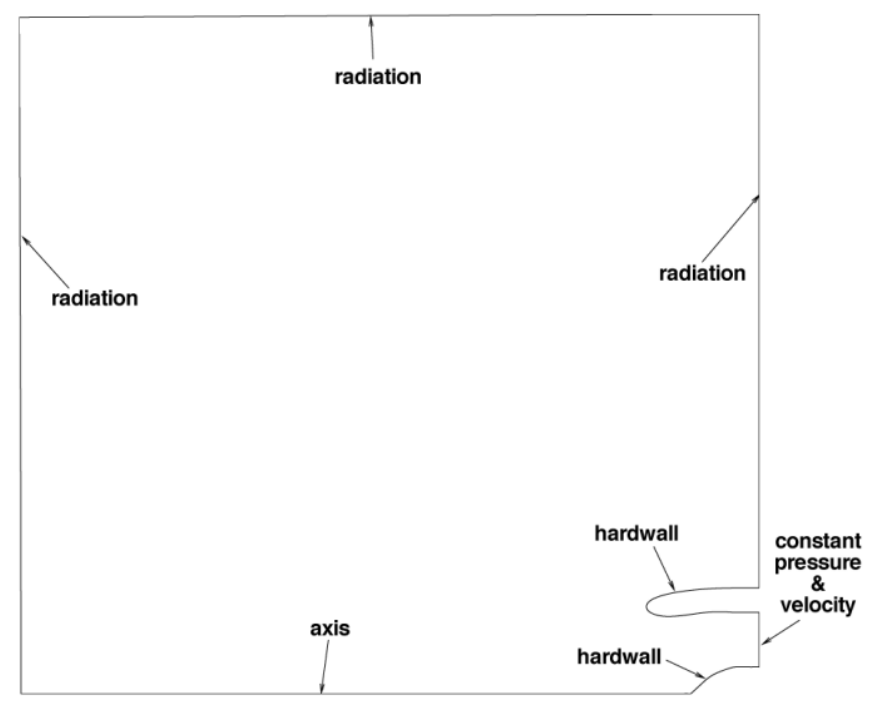

Figure 11. Boundary conditions imposed in the numerical simulations of mean flow.

In addition to the governing equations, boundary conditions are needed to compute the mean flow. The different types of boundary conditions imposed in the present computation are shown in Fig. 11. The radiation boundary conditions used are the asymptotic radiation boundary conditions developed in Ref. [30]. Because these conditions are derived from asymptotic solutions, the equations implicitly require the specification of a center of acoustic source. The radiation boundary conditions may be written as,

$$
\left[\frac{1}{V(\theta)} \frac{\partial}{\partial t}+\cos \theta \frac{\partial}{\partial x}+\sin \theta \frac{\partial}{\partial r}+\frac{1}{\sqrt{\left(x-x_{s}\right)^{2}+\left(r-r_{s}\right)^{2}}}\right]\left[\begin{array}{c}
\rho-1 \\
u-M_{\text {fight }} \\
v \\
p-\frac{1}{\gamma}
\end{array}\right]=0
$$

where $M_{\text {fight }}$ is the forward flight Mach number, $\left(x_{s}, r_{s}\right)$ are the physical coordinates of the sound source and $\theta$ is the angle between the positive $x$-axis and the line connecting the source point and the boundary point. In addition, $V(\theta)$, the asymptotic acoustic wave speed, is given by,

$$
V(\theta)=\left[M_{\text {fight }} \cos \theta+\left(1-M_{\text {flight }}^{2} \sin ^{2} \theta\right)^{1 / 2}\right]
$$

The acoustic source location to be used for determining angle $\theta$ in Eqs. (5) and (6) is shown in Fig. 3. For boundary $\mathrm{ABC}$, the origin is at the point $\mathrm{O}$. For boundary $\mathrm{CDE}$, the source is taken to lie along the line segment $\mathrm{OM}$, with the source at point $\mathrm{O}$ for boundary point $\mathrm{C}$ and the source at point $\mathrm{M}$ for boundary point $\mathrm{E}$. For intermediate boundary points along $\mathrm{CDE}$, the source point distance from $\mathrm{O}$ along $\mathrm{OM}$ is kept proportional to the boundary point distance from $\mathrm{C}$ along $\mathrm{CDE}$. For boundary $\mathrm{EF}$, the origin is taken to be point $\mathrm{M}$. 
For the axis boundary condition, the method of Ref. [31] is used. This set of boundary conditions avoids the apparent singularity at the axis of the cylindrical coordinates where $r=0$. This axis boundary treatment works well in all the present computations. At the fan face boundary, the desired Mach number is prescribed. The corresponding value of pressure is computed according to conservation of enthalpy and is enforced at the boundary.

\subsection{Acoustic computation}

The acoustic field in the computational domain is governed by the linearized Euler equations. They can be derived easily. For this reason, these equations will not be written out explicitly. The various types of boundary conditions to be imposed on the acoustic field are shown in Fig. 12. They are almost the same as those for the mean flow computation. The exception is at the fan face boundary. Here, an incoming duct mode is introduced into the computational domain. For this purpose, the split variable method (see Ref. [32]) is used. To absorb any possible acoustic disturbances reflected back to the fan face from the interior of the computational domain, a Perfectly Matched Layer (PML) absorbing boundary condition is added externally to the computational domain. The PML formulation and implementation follow the work of Ref. [33, 34]. We find computationally that this boundary treatment performs satisfactorily.

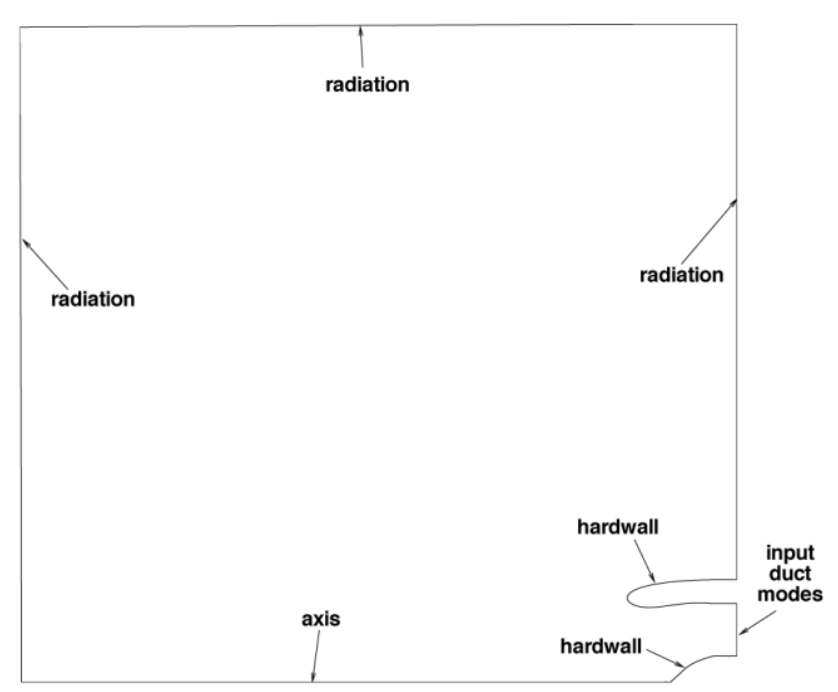

Figure 12. Boundary conditions used to compute the acoustic field.

All time marching computations in the present investigation are carried out using the multi-size-mesh multi-time-step DRP scheme of Ref. [28]. This scheme has proven to be accurate and efficient.

\subsection{Acoustic duct modes}

It will be assumed that the sound to be radiated out of the engine inlet is from a duct mode of an annular duct with a hub-tip ratio $\sigma$. The fan face Mach number and the forward flight Mach number, or wind tunnel Mach number in the case of simulated forward flight, are denoted by $M_{\text {fan }}$ and $M_{\text {flight }}$, respectively. For a duct mode with azimuthal mode number $m$ and radial mode number $n$, the dimensionless axial wave number $k_{m n}$ (for simulated forward flight) is,

$$
k_{m n}=\frac{-\omega u_{f}-\left\{\left[\frac{1+\frac{\gamma-1}{2} M_{\text {fight }}^{2}}{1+\frac{\gamma-1}{2} M_{\mathrm{fan}}^{2}}\right] \omega^{2}-\left(\left[\frac{1+\frac{\gamma-1}{2} M_{\text {flight }}^{2}}{1+\frac{\gamma-1}{2} M_{\text {fan }}^{2}}\right]-u_{f}^{2}\right)\left[\frac{1+\frac{\gamma-1}{2} M_{\text {fight }}^{2}}{1+\frac{\gamma-1}{2} M_{\mathrm{fan}}^{2}}\right] \beta_{m n}^{2}\right\}^{\frac{1}{2}}}{\left[\frac{1+\frac{\gamma-1}{2} M_{\text {flight }}^{2}}{1+\frac{\gamma-1}{2} M_{\mathrm{fan}}^{2}}\right]-u_{f}^{2}}
$$


where $\omega$ is the angular frequency, $u_{f}$ is the fan face velocity given by,

$$
u_{f}=M_{f}\left[\frac{1+\frac{\gamma-1}{2} M_{\text {flight }}^{2}}{1+\frac{\gamma-1}{2} M_{\text {fan }}^{2}}\right]^{\frac{1}{2}}
$$

and $\beta_{m n}$ is the root of the dispersion relation,

$$
J_{m}^{\prime}\left(\frac{1}{2} \beta_{m n}\right) Y^{\prime}\left(\frac{\sigma}{2} \beta_{m n}\right)-Y_{m}^{\prime}\left(\frac{1}{2} \beta_{m n}\right) J_{m}^{\prime}\left(\frac{\sigma}{2} \beta_{m n}\right)=0
$$

$J_{m}()$ and $Y_{m}()$ are the Bessel and Neumann functions of order $m$. The duct mode eigenfunction is,

$$
\left[\begin{array}{c}
p_{m n} \\
u_{m n} \\
v_{m n} \\
w_{m n} \\
\rho_{m n}
\end{array}\right]=A_{m n}\left[\begin{array}{c}
1 \\
\frac{k_{m n}}{\rho_{\mathrm{fan}}\left(\omega-k_{m n} u_{f}\right)} \\
\frac{-i \beta_{m n}}{\rho_{\mathrm{fan}}\left(\omega-k_{m n} u_{f}\right)} \\
\frac{m}{\rho_{\mathrm{fan}}\left(\omega-k_{m n} u_{f}\right)} \\
\frac{1+\frac{1}{2}(\gamma-1) M_{\mathrm{fan}}^{2}}{1+\frac{1}{2}(\gamma-1) M_{\mathrm{flight}}}
\end{array}\right]\left\{J_{m}\left(\beta_{m n} r\right)-\frac{J_{m}^{\prime}\left(\frac{\sigma}{2} \beta_{m n}\right)}{Y_{m}^{\prime}\left(\frac{\sigma}{2} \beta_{m n}\right)} Y_{m}\left(\beta_{m n} r\right)\right\} e^{i\left(k_{m n} x+m \phi-\omega t\right)}
$$

Note: In this work, the radial mode number $n$ is set equal to the total number of maxima and minima of the eigenfunction. According to this numbering system, $n=0$ is possible only for the plane wave mode (i.e. $m=n=0)$. For azimuthal modes with $m \neq 0$, the lowest order radial mode is $n=1$.

Cantrell and $\mathrm{Hart}^{35}$ derived an expression for the energy flux in the upstream direction. On following their formula, with fan face variables $\rho_{f}, u_{f}, a_{f}$, the energy flux, $F_{m n}$, for the $(m, n)$ duct mode is found to be,

$$
F_{m n}=\pi Q_{m n} A_{m n}^{2}\left\{\left[1+u_{f}\left(\frac{1+\frac{\gamma-1}{2} M_{\mathrm{fan}}^{2}}{1+\frac{\gamma-1}{2} M_{\mathrm{flight}}^{2}}\right)\right] \frac{k_{m n}}{\rho_{f}\left(\omega-k_{m n} u_{f}\right)}+\frac{u_{f}}{\rho_{f}}\left[\frac{k_{m n}^{2}}{\left(\omega-k_{m n} u_{f}\right)^{2}}+\frac{1}{a_{f}^{2}}\right]\right\}
$$

where

$$
Q_{m n}=\left\{\begin{array}{c}
\frac{1}{8}\left(1-\sigma^{2}\right), \quad m=n=0, \beta_{m n}=0 \\
\int_{\sigma / 2}^{1 / 2}\left[J_{m}\left(\beta_{m n} r\right)-\frac{J_{m}^{\prime}\left(\beta_{m n} \frac{\sigma}{2}\right)}{Y_{m}^{\prime}\left(\beta_{m n} \frac{\sigma}{2}\right)} Y_{m}\left(\beta_{m n} r\right)\right]^{2} r d r, \beta_{m n} \neq 0
\end{array}\right.
$$

If PWL is the sound power level of the $(m, n)^{\text {th }}$ duct mode in $\mathrm{dB}$ and $W_{0}=10^{-12}$ watts is the reference sound power level for the $\mathrm{dB}$ unit, the duct mode amplitude can easily be found to be,

$$
A_{m n}=\left\{\frac{W_{0} 10^{\mathrm{PWL} / 10}}{\rho_{0} a_{0}^{3} D^{2} \pi Q_{m n}\left[1+u_{f}^{2}\left(\frac{1+\frac{\gamma-1}{2} M_{f}^{2}}{1+\frac{\gamma-1}{2} M_{0}^{2}}\right)\right] \frac{k}{\rho_{f}\left(\omega-k u_{f}\right)}+\frac{u_{f}}{\rho_{f}}\left[\frac{k^{2}}{\left(\omega-k u_{f}\right)^{2}}+\frac{1}{a_{f}^{2}}\right]}\right\}^{\frac{1}{2}}
$$




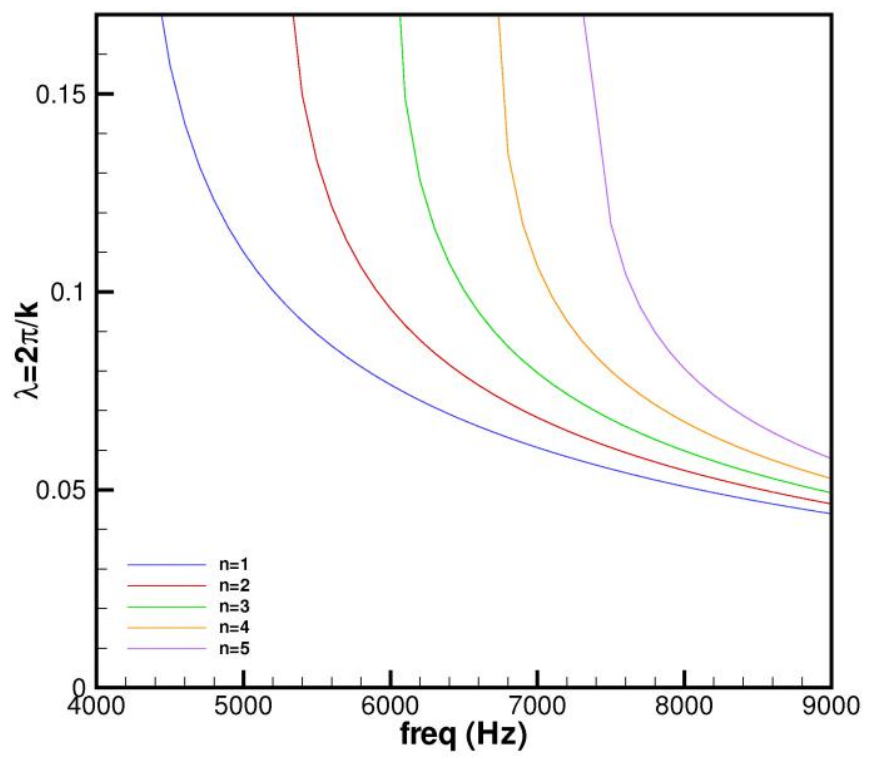

Figure 13. Variation of duct mode axial wavelength with frequency. $M_{\text {fan }}=0.4, M_{\text {flight }}=0, m=22$.

Of interest later on is the dependence of the axial wavelengths of duct modes on frequency and azimuthal mode number. The axial wavelength of the $(m, n)^{\text {th }}$ mode, $\lambda_{m n}$, is related to the axial wave number $k_{m n}$ of Eq. (7) by $\lambda_{m n}=2 \pi / k_{m n}$. Thus by examining the numerator of Eq. (7), it is easy to see that for fixed values of $M_{\text {fan }}, M_{\text {flight }}, m$ and $n$, an increase in frequency leads to an increase in $k_{m n}$ and hence a decrease in wavelength. Fig. 13 shows typical examples of the reduction in axial wavelength with increase in frequency.

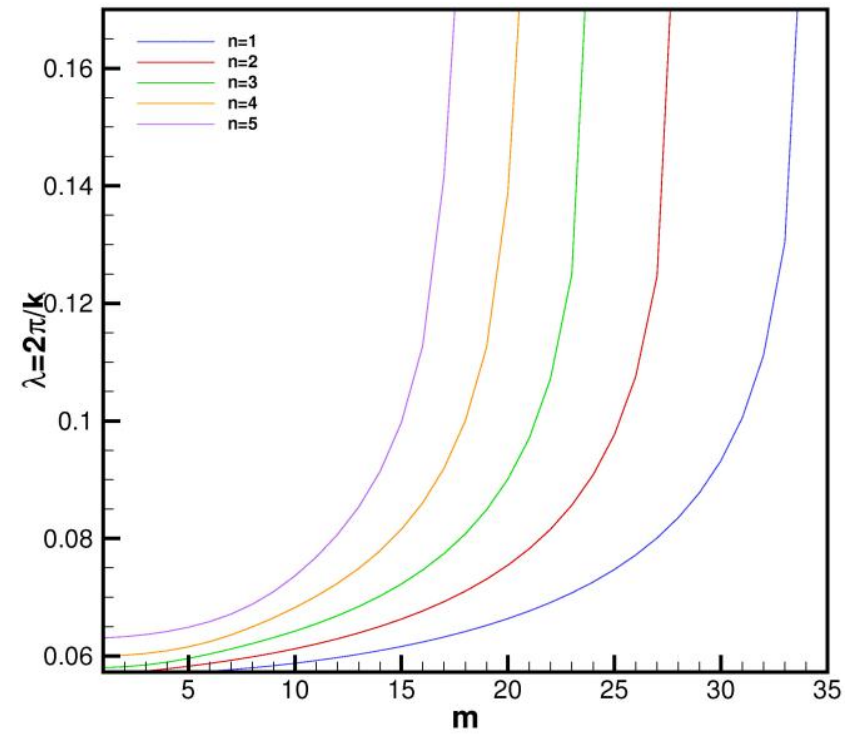

Figure 14. Variation of duct mode axial wavelength with azimuthal mode number.

$$
M_{\text {fan }}=0.4, M_{\text {flight }}=0, f=6400 \mathrm{~Hz}
$$

The eigenvalue $\beta_{m n}$ of Eq. (7) is the root of Eq. (8). It is well known that for a fixed radial mode number $n$, the value of $\beta_{m n}$ from Eq. (8) increases with $m$. Because of this, it follows from Eq. (7) that for fixed $M_{\text {fan }}, M_{f i g h t}$, 
$n$ and frequency $f$ or $\omega$, a decrease in azimuthal mode number $m$ would lead to an increase in $k_{m n}$ and, therefore, a reduction in axial wavelength. Fig. 14 shows typical examples of the variation of $\lambda_{m n}$ with $m$.

\subsection{Extension to the far field}

A computational domain inevitably has a finite size. To determine far field directivity, it is, therefore, necessary to extend the numerical solution to the far field. Currently, the Kirchhoff method and the Ffowcs Williams and Hawkings method ${ }^{36}$ (see also Pilon and Lyrintzis ${ }^{37}$ and Lyrintzis ${ }^{38}$ ) are the two most popular methods for extending near field results to the far field. To use these methods, one encloses the near field by a matching surface. The extension is to continue the information prescribed on the matching surface to the far field. In both the Kirchhoff method and the Ffowcs Williams and Hawkings method, at least three sets of data are required on the matching surface. The data needed are the pressure, the normal gradient of the pressure on the surface and the time derivative of pressure. Recently, Reba, Simonich and Schlinker ${ }^{39}$, Reba, Narayanan, Colonius and Suzuki ${ }^{40}$ and Tam, Pastouchenko and Viswanathan ${ }^{41}$ have shown that the near-to-far acoustic field extension, or the continuation problem, can also be accomplished by the use of a surface Green's function. For the jet engine inlet duct mode radiation problem, it turns out the surface Green's function method is the simplest to use and to implement. Instead of requiring three sets of data as the Kirchhoff method and the Ffowcs Williams and Hawkings method, the surface Green's function method requires only one set of data, namely, the value of pressure on the matching surface. For this reason, the surface Green's function method is adopted in this work. The mathematical details of the surface Green's function method for the jet engine inlet noise radiation problem are given in Appendix A.

\section{Code Validation}

To validate the computer code developed in this investigation, the JT15D static engine test data is used. Details of this experiment can be found in the papers by Heidelberg, Rice and Homyak ${ }^{36}$, Baumeister, and Horowitz $^{14}$, and Preisser, Silcox, Eversman, and Parrett ${ }^{2}$. This set of data has been used for code validation by Ozyoruk, Alpman, Ahuja, and Long ${ }^{24}$. Fig. 15 shows the geometry of the JT15D engine inlet. In the experiment, the inlet was first tested with a hard wall surface. Then a liner segment was inserted in the casing, as shown in Fig. 15, and the liner configuration was tested for two separate values of impedance. Hence, three sets of experimental data are available for validation.

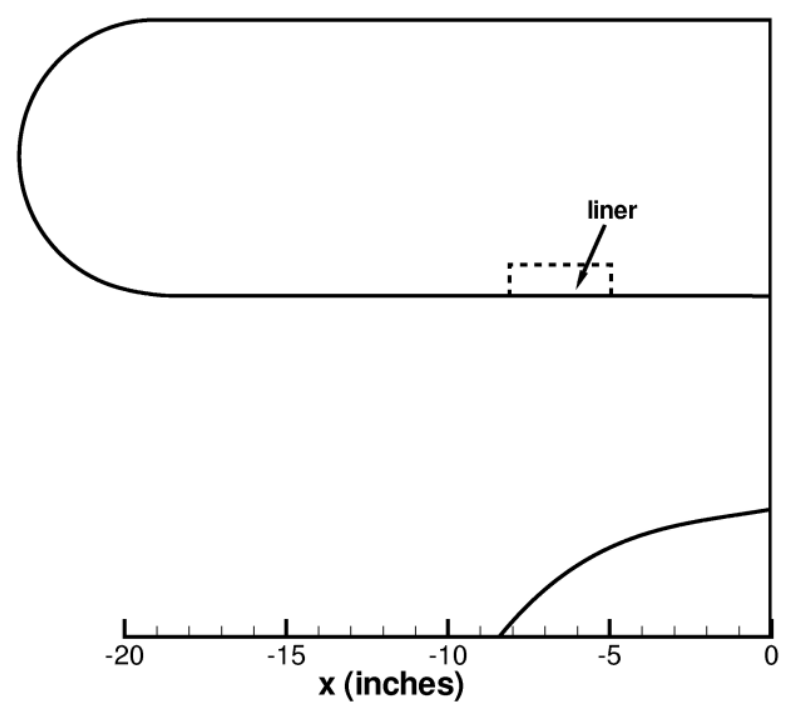

Figure 15. Geometry of JT15D engine inlet, including the location of a liner segment.

The fan of the JT15D engine has 28 blades operating at a blade passage frequency of $3150 \mathrm{~Hz}$. The fan face Mach number is 0.147 . There are 41 small rods mounted on the casing wall ahead of the fan. The wake shed by the rods is cut by the fan blades, creating strong interaction tones. By design, the inlet geometry and the blade 
passage frequency support only one propagating mode with $m=13$ and $n=1$. The fact that there is only one propagating mode greatly simplifies the effort to compare numerical results with experimentally measured directivities.

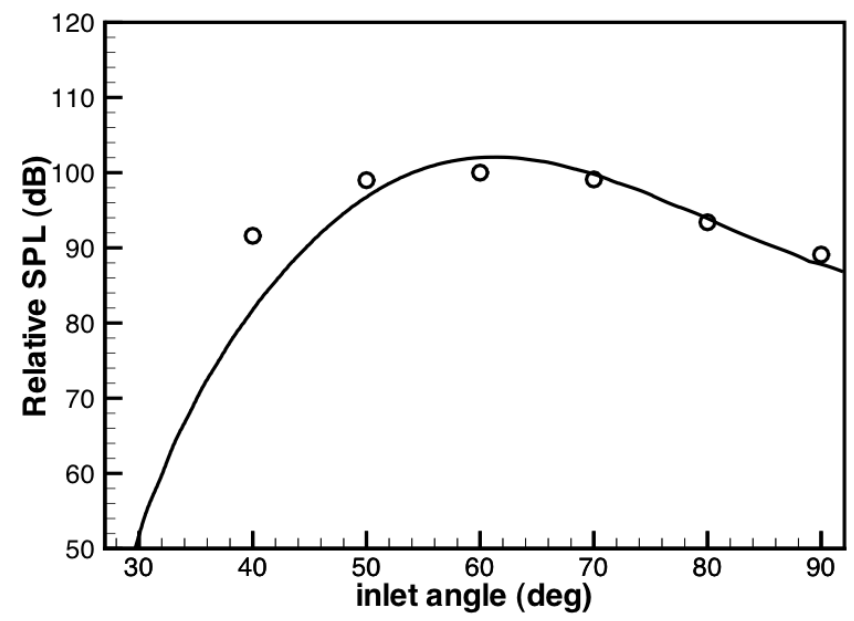

Figure 16. Comparison between computed and measured directivity (hard wall).

- computed, $O$ measured.

Fig. 16 shows a comparison between the experimentally measured and computed directivities for the hard wall inlet. As can be seen, there is a reasonably good overall agreement. The degree of agreement is comparable to that obtained by Ozyoruk, Alpman, Ahuja and Long ${ }^{24}$. Figs. 17 and 18 are comparisons of measured and computed relative directivities for the cases with a liner. In Fig. 17 the impedance of the liner is $\mathrm{Z}=0.638+i 0.5$. Fig. 18 shows the comparison for a liner with impedance $Z=1.136+i 0.5$. There are good to reasonably good overall agreements in these cases. Again, the degree of agreement is similar to that obtained by Ozyoruk et al ${ }^{24}$.

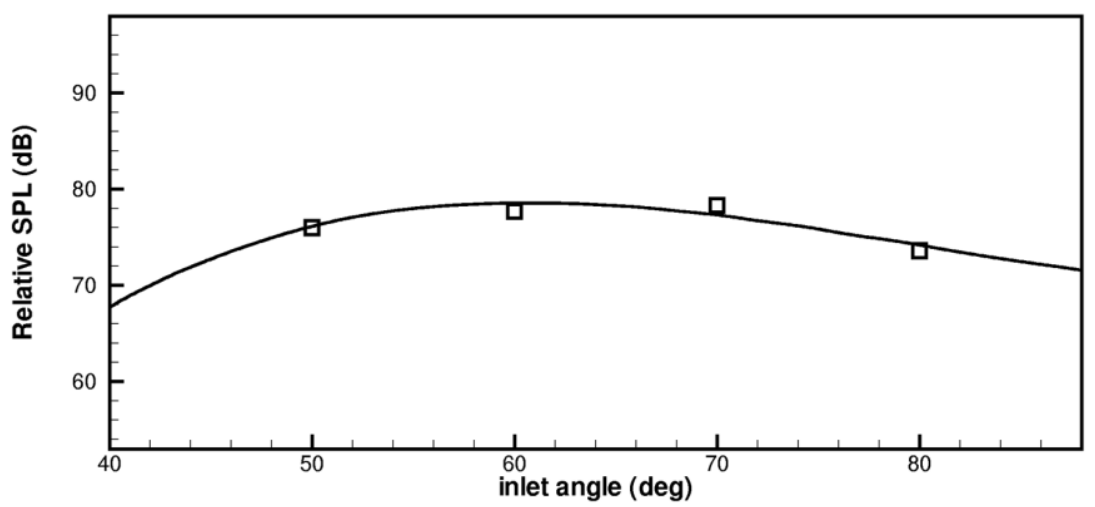

Figure 17. Comparison between computed and measured directivity (with liner). Liner impedance $Z=0.638+i 0.5$. - computed, $\square$ measured. 


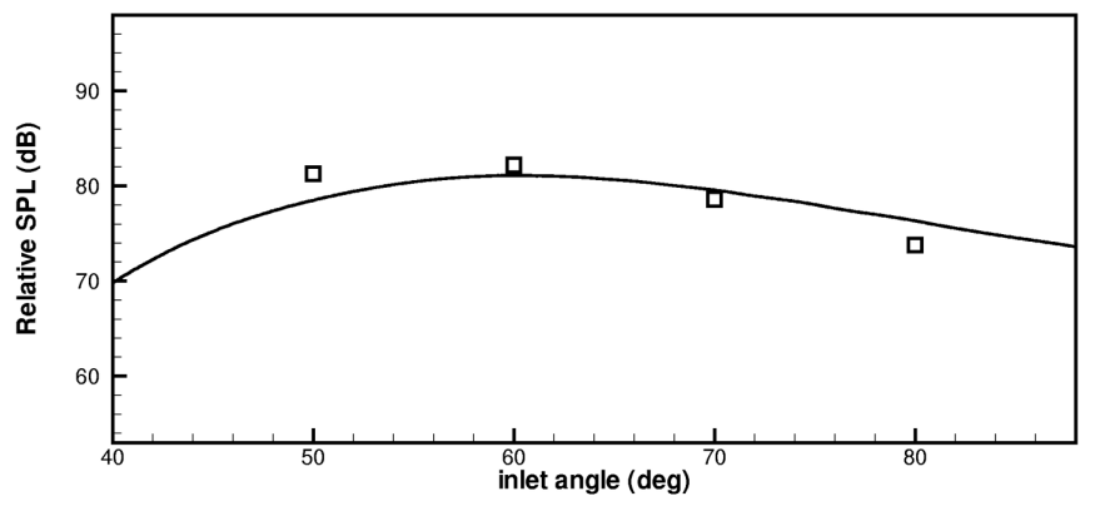

Figure 18. Comparison between computed and measured directivity (with liner). Liner impedance $Z=1.136+i 0.5$.

computed, $\square$ measured.

\section{Mean Flow}

The mean flow around the inlet of a jet engine changes drastically when there is forward flight. In order to highlight these changes, a typical mean flow computed at static condition will first be presented. This is to be followed by a mean flow computation with a low forward flight Mach number but with the same fan face Mach number.

\subsection{Mean flow at static condition}

Fig. 19 shows the streamline pattern around the SDT fan inlet at a fan face Mach number of 0.4. The streamlines are bunched together near the tip of the casing. This indicates that the flow is at a high speed around the casing tip. Fig. 20 is an enlarged streamline pattern focusing on the inside of the engine inlet.

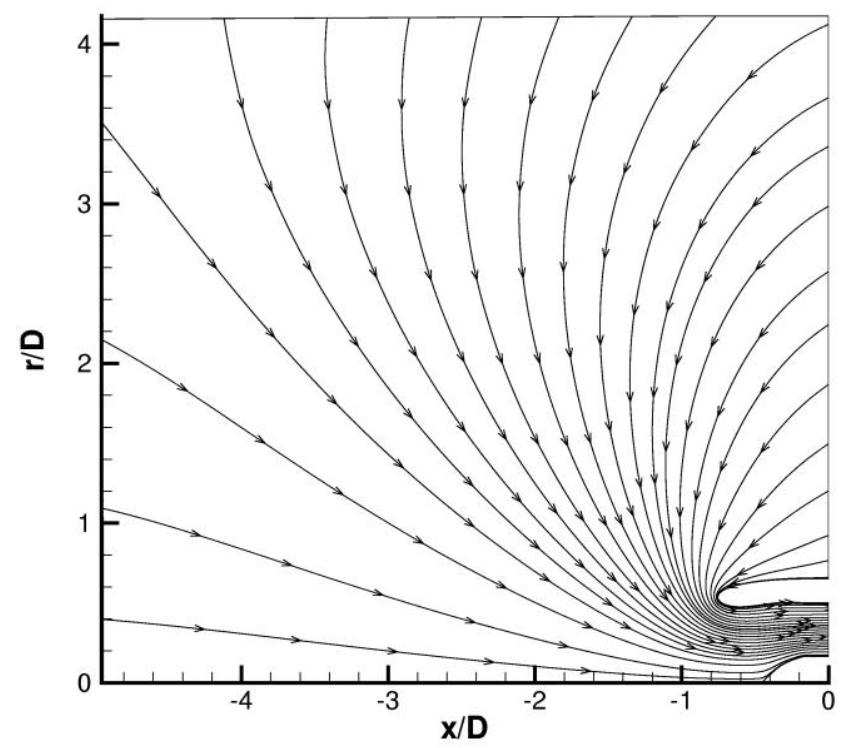

Figure 19. Computed streamline pattern at the inlet of the SDT engine at static condition.

Fan face Mach number $=0.4$. 


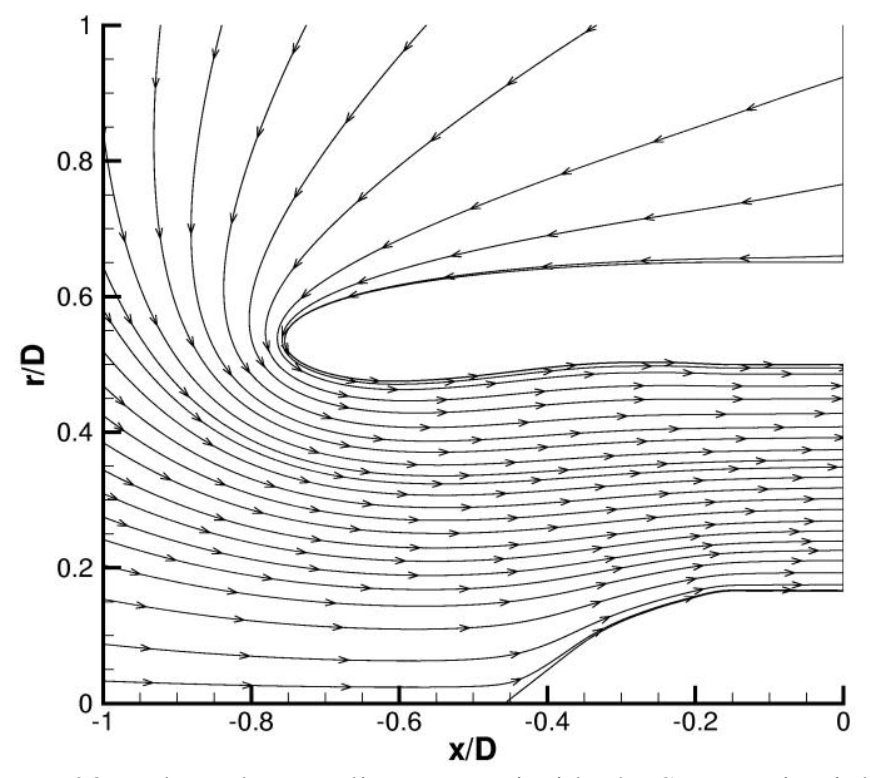

Figure 20. Enlarged streamline pattern inside the SDT engine inlet.

Fig. 21 plots the tangential velocity, $V_{\text {tan }}$, along the casing wall. Fig. 21a shows the locations of selected points $\mathrm{A}, \mathrm{B}, \mathrm{C}, \mathrm{D}, \mathrm{E}$, and $\mathrm{F}$ along the casing wall. Fig. $21 \mathrm{~b}$ provides a plot of the tangential velocity on the casing versus the radial distance from the axis of the inlet. At point A, the flow velocity is practically zero, which is the ambient condition. From A to D the flow velocity accelerates quickly to a Mach number of 0.74 . This is a transonic Mach number. It is almost twice the fan face Mach number. Along DEF the flow velocity decelerates. It reduces further to Mach 0.4 at the fan face.

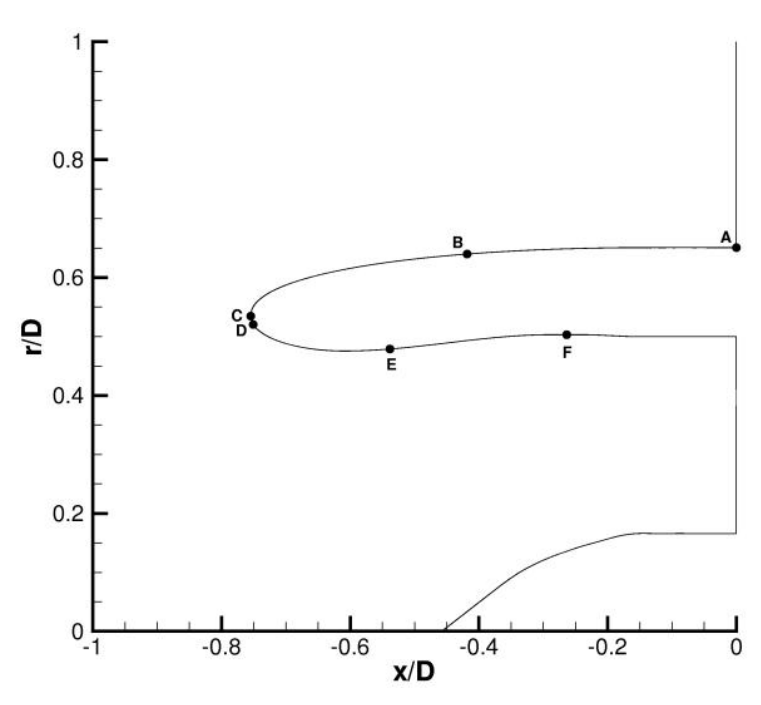

Fig. 21a. Location of points A,B,C,D,E, and F on casing.

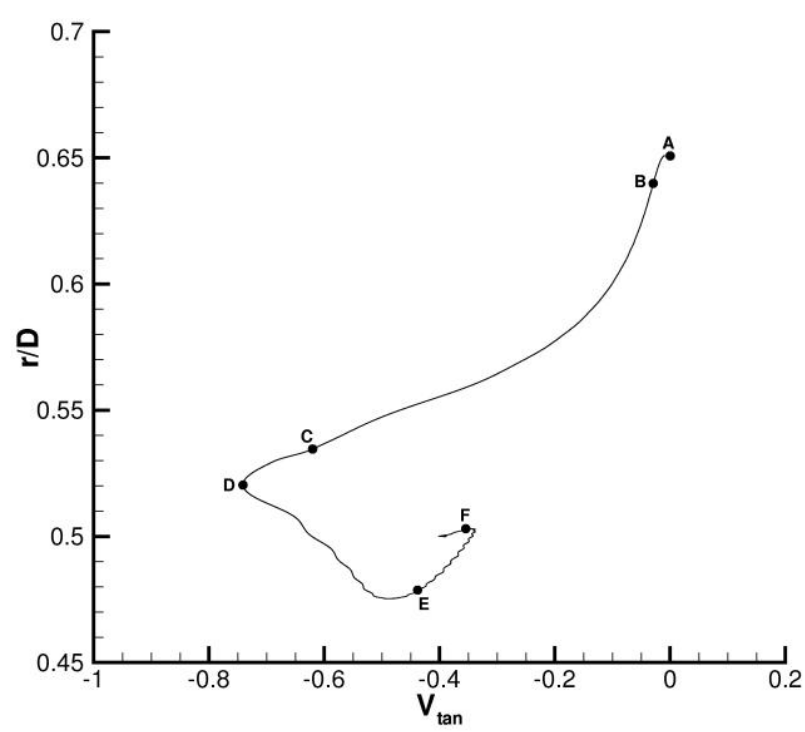

Fig. 21b. Tangential velocity at A,B,C,D,E, and F.

Fig. 22 shows that the mean flow reaches its highest velocity at the lip of the casing. The flow is slower at the center of the inlet. As a result, a significant velocity gradient develops as indicated in this figure. The velocity gradient points towards the lip of the casing, toward the point with the highest velocity. The arrow in this figure points in the direction of negative gradient. 


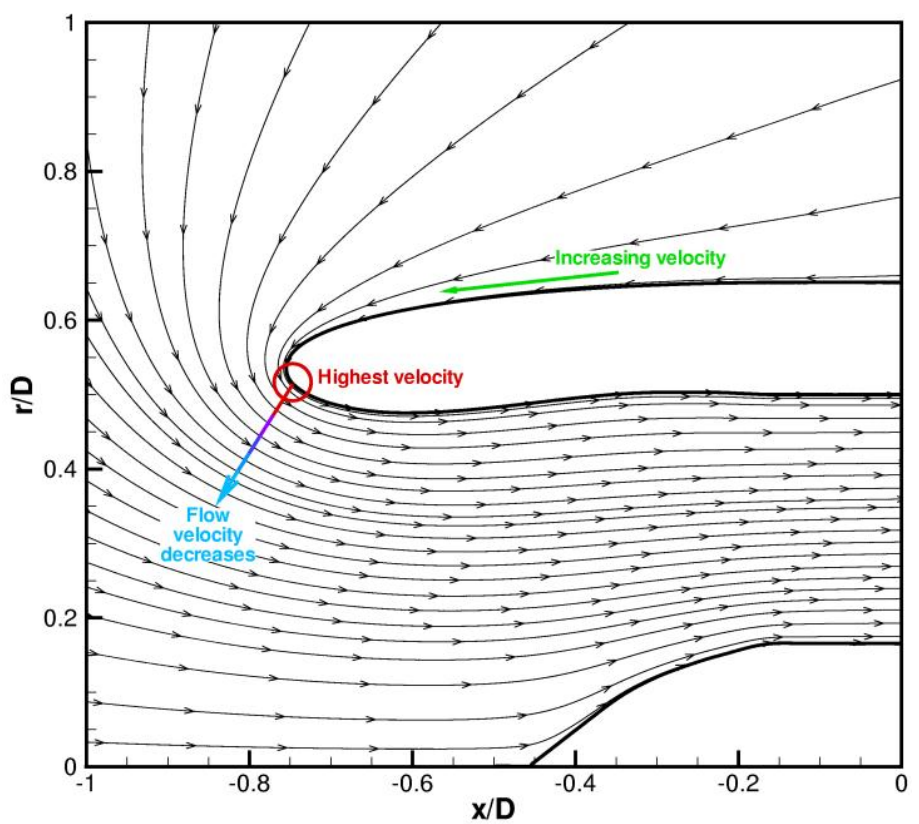

Figure 22. Strong velocity gradient develops near the lip of the casing. Arrow points in the direction of negative gradient.

\subsection{Mean flow in forward flight}

Fig. 23 shows the mean flow streamline pattern at the same fan face Mach number as Fig. 19 but now there is an external flow at Mach 0.2. The streamline patterns of the two figures are drastically different. With forward flight, only the flow in a stream tube bounded by the separation streamline is ingested by the engine. The separation streamline is shown as a thick dark line in this figure. The separation streamline terminates at the lip of the casing. Outside the separation streamline, the fluid simply flows past the engine.

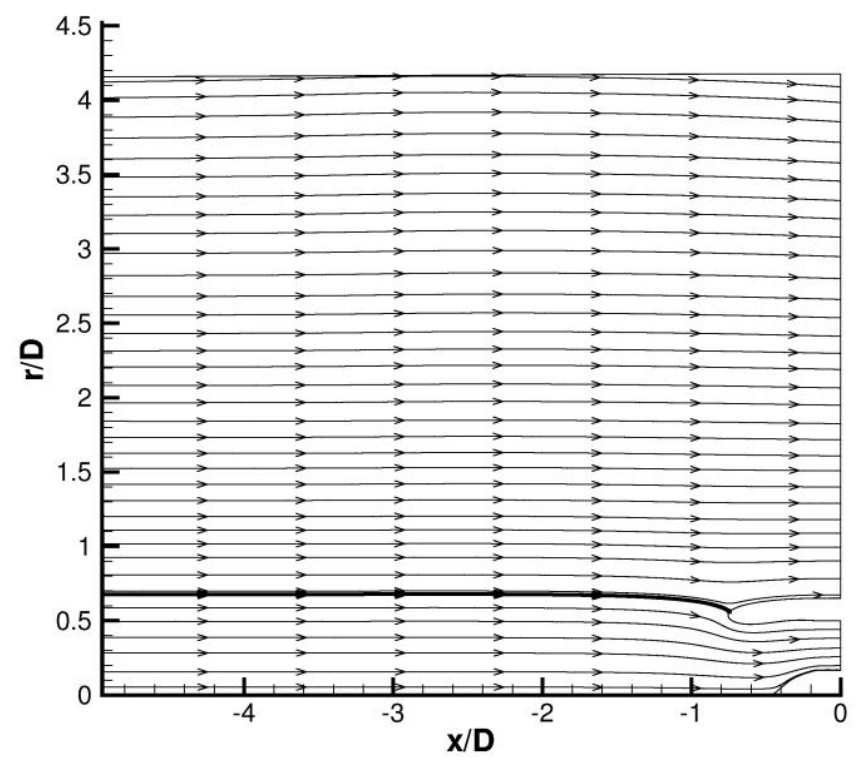

Figure 23. Streamline pattern around the SDT engine inlet at fan face Mach number 0.4 and forward flight Mach number 0.2 .

Fig. 24 shows an enlarged streamline pattern around the SDT engine inlet. The point at which the separation streamline terminates at the casing lip is a stagnation point. The flow decreases in speed towards the 
stagnation point. On passing this point on the casing wall, the flow starts to accelerate and ultimately reaches Mach 0.4 at the fan face. Now, around the lip of the casing the flow velocity is the lowest at the stagnation point. This creates a velocity gradient pointing away from the casing wall. It is to be noted that the direction of the velocity gradient is opposite in the case with no forward flight, as shown in Fig. 22. This contrast in the direction of the velocity gradient for static and flight conditions turns out to be rather significant, as will be seen in the following section.

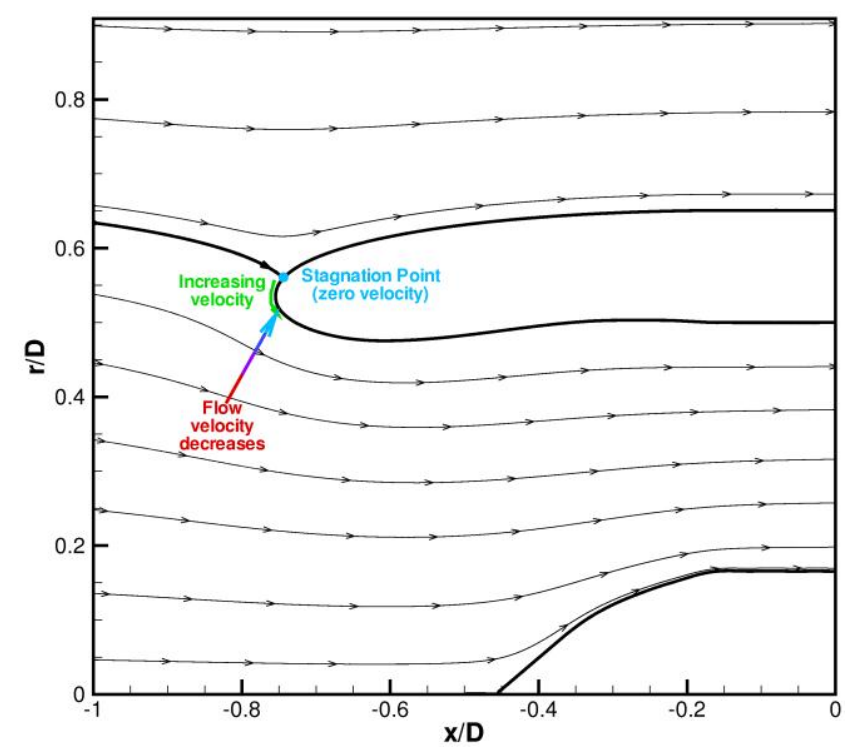

Figure 24. Mean velocity gradient developed near the lip of the casing at forward flight Mach number 0.2.

\section{Diffraction and Refraction}

In this section, duct mode radiation patterns and directivities computed with and without forward flight are presented. The directivities with forward flight are quite different from those without. The reason for this difference will be explained below. Two effects are most important in affecting the direction of radiation. They are: diffraction and refraction. Essentially, the combination of these two effects determines the radiation pattern of a duct mode.

\subsection{Diffraction}

In the context of duct mode radiation, we will define diffraction as the natural tendency of the pressure field of a duct mode to follow a curved solid surface as it propagates. This effect causes the wave field's direction of propagation to make a large turn at the lip of an engine casing. To illustrate this process, consider a duct mode of $m$ $=22, n=1$ at a frequency of $6.4 \mathrm{kHz}$ radiating out of the SDT engine inlet. We will first study the case without mean flow. That is, the fan face Mach number is zero. This allows us to investigate the propagation of duct modes along the casing wall without any modification by the mean flow. Fig. 25 shows the radiation pattern. The lines are pressure contours. In this figure, red is high pressure and blue is low pressure. The duct mode does not propagate straight out of the inlet in the forward direction. At the casing lip, the sound field turns at an angle of about 45 degrees before radiating away. 


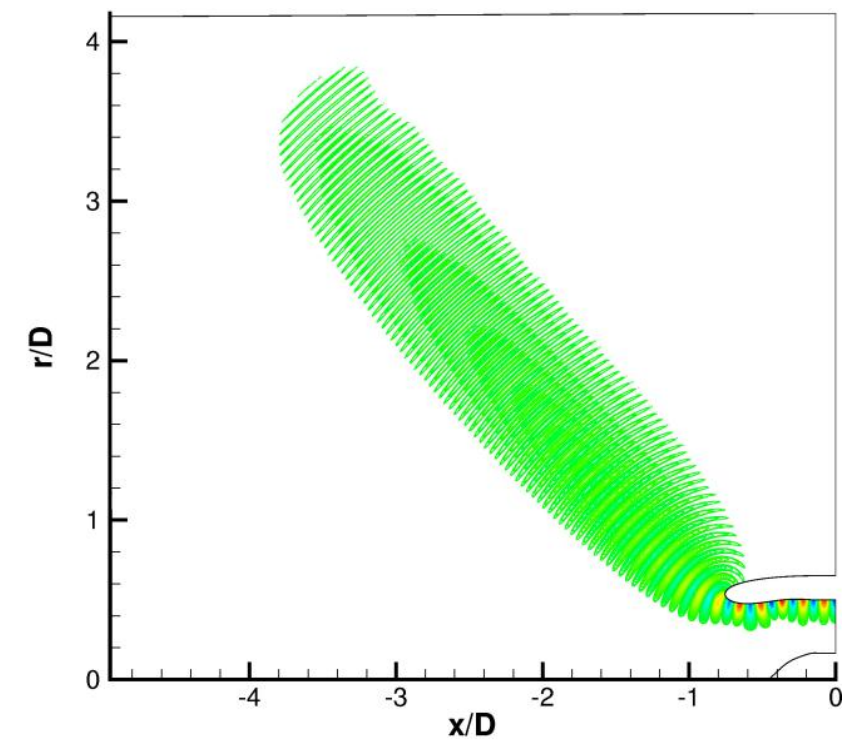

Figure 25. Near field pressure contour pattern showing the dominant direction of radiation.

No mean flow. Duct mode has $m=22, n=1$.

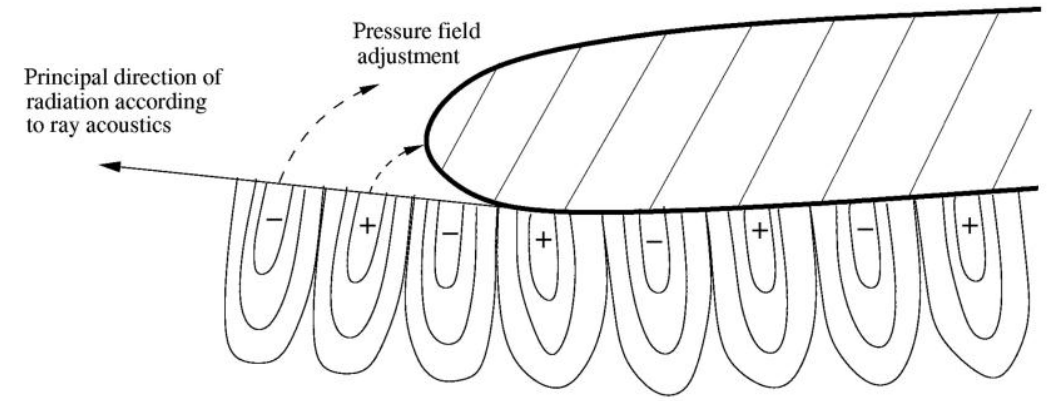

Figure 26. A schematic sketch illustrating the complete separation of a duct mode from the casing wall when radiating out of an engine inlet. Required pressure balance prevents this type of complete separation.

Now, a duct mode is made up of alternating high and low pressure regions. The highest and the lowest pressure points are right next to the wall, as shown in Figs. 25 and 26. When the sound field propagates along a solid surface, the surface can sustain the high and low pressure variations. Suppose the duct mode propagates in a strictly forward direction, separating from the casing surface as shown in Fig. 26. Immediately, there would be a pressure imbalance along the line of separation because the ambient pressure is constant. Thus, the pressure field of the duct mode must adjust its pressure distribution by shifting the highest and lowest pressure points away from the wall while it remains attached to the wall, as shown in Fig. 27a. Fig. 27b shows a diffraction pattern (pressure contours) at $6400 \mathrm{~Hz}$ for a duct mode with $m=22$ and $n=1$. The attachment of the sound field to the casing wall is clearly shown. 


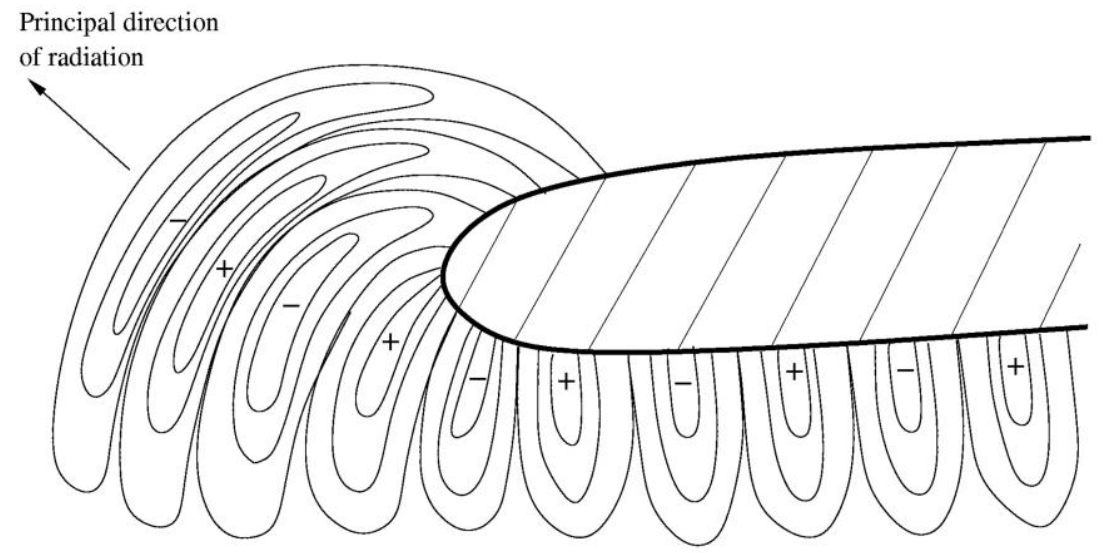

Figure 27a. To maintain pressure balance, a radiating duct mode must adjust its pressure distribution and remain attached to the casing wall. The adjustment causes the direction of propagation to rotate in the clockwise direction.

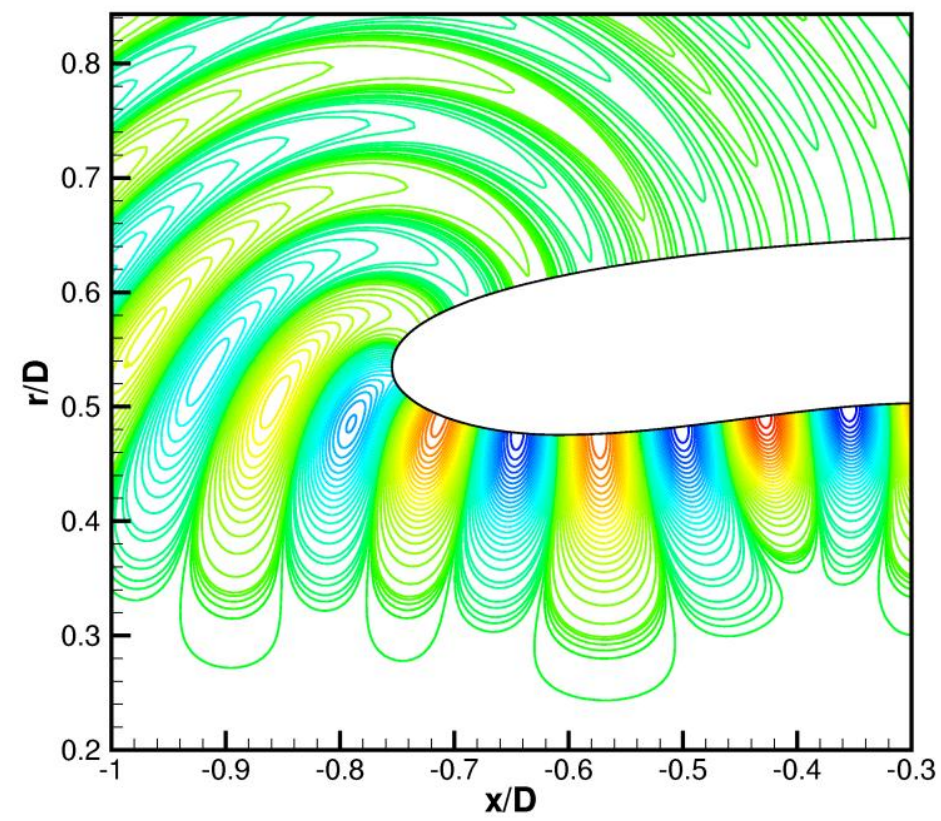

Figure 27b. Instantaneous distribution of pressure contours associated with the radiation of a duct mode with $m=22$ and $n=1$ at $6400 \mathrm{~Hz}$ from the SDT engine inlet.

Diffraction causes the pressure field to rotate in the clockwise direction. As the duct mode turns, the highest and lowest pressure regions gradually move away from the wall. When the highest and lowest pressure regions have detached sufficiently from the wall, the sound field can then radiate away. These processes are observable in a highspeed animation. Thus, the effect of diffraction is what leads the duct mode in Fig. 25 to radiate predominantly around the $45^{\circ}$ direction.

The effect of diffraction naturally depends on the axial wavelength of the duct mode. For duct modes with short wavelengths, the diffraction effect is small. This should become clear if one considers the limit of very small wavelengths. In this limit, the sound waves are rays, like that of light. If light waves were to radiate out of an engine inlet, they would mostly radiate in the forward direction. In other words, the diffraction effect is absent or minimal. What this reasoning suggests is that the diffraction effect is stronger for long waves and less significant for short waves. Fig. 28 shows the diffraction pattern of duct modes with $m=22$ and $n=1$ at a range of frequencies from 5.2 $\mathrm{kHz}$ to $7.6 \mathrm{kHz}$. It is obvious that at low frequencies the axial wavelength is longer (see Fig. 13). For these long waves, the turning angle of the direction of radiation (clockwise) measured from the inlet direction is larger. This is consistent with the above deduction. Fig. 29 is a symbolic representation of the dependence of the turning angle on 
wavelength. In this figure, $\lambda_{1}>\lambda_{2}>\lambda_{3}$ implies decreasing wavelength. The radius of the arc of rotation and the thickness of the arc in the figure represent the relative size of the angle of rotation. The point is: the shorter the wavelength, the smaller the clockwise turning angle from diffraction.

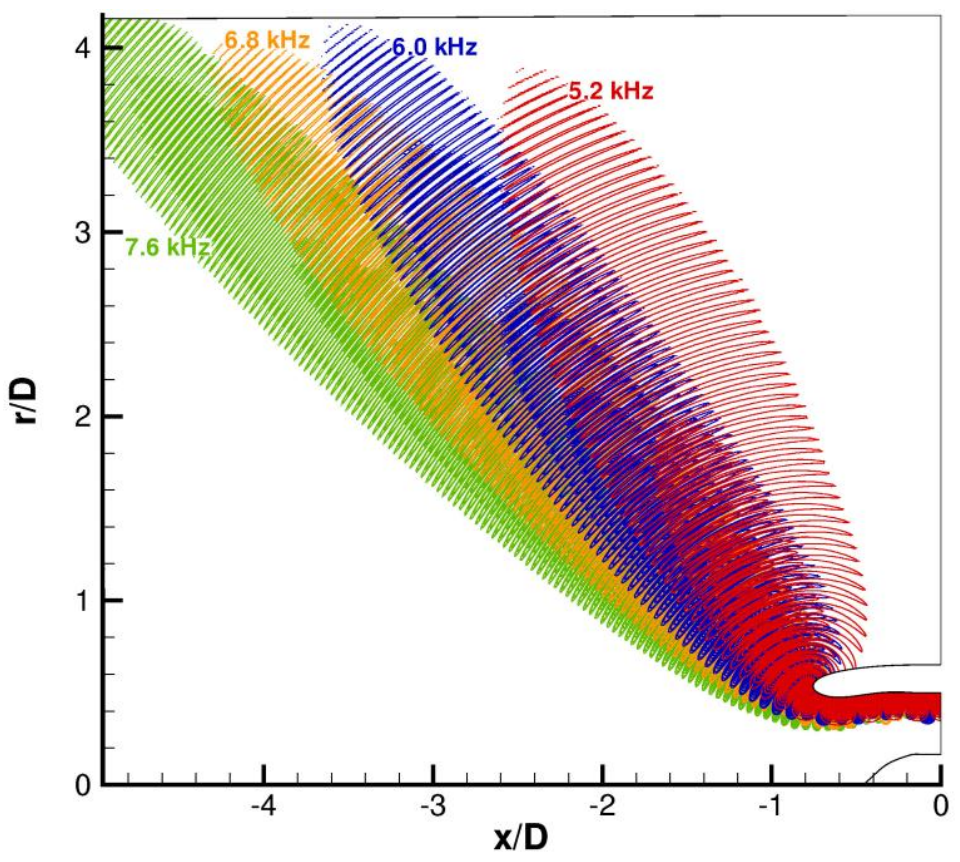

Figure 28. Pressure contour patterns showing the radiation of duct modes from the SDT engine inlet (without mean flow) at a number of frequencies. All duct modes have respective azimuthal and radial mode numbers of $m=22$ and $n=1$.

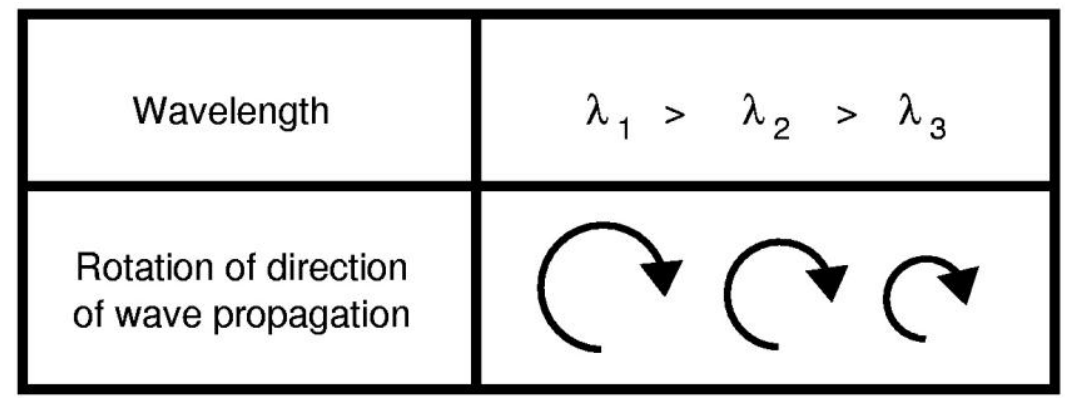

Figure 29. Symbolic representation of the dependence of diffraction effect on duct mode wavelength.

\subsection{Refraction}

A jet engine draws in a large quantity of air at its inlet. As discussed in the previous section, this creates a nonuniform mean flow with a significant velocity gradient around the casing lip. At a fan face Mach number of 0.4 , in static condition, the mean flow causes the radiation pattern to change from Fig. 25 to Fig. 1. Fig. 30 is a superposition of the two radiation patterns. It is clear that the presence of a mean flow causes the dominant direction of radiation to rotate in the clockwise direction almost an additional $45^{\circ}$ past the angle caused by diffraction only. This substantial change in the radiation direction is due to the effect of refraction. At static condition, the velocity gradient near the casing lip is shown in Fig. 22. Now consider a wave front $\mathrm{AA}^{\prime}$ as shown in Fig. 31. The wave is propagating against the flow. The flow velocity is highest along the casing wall $\mathrm{AB}$ and much slower along streamline $\mathrm{A}^{\prime} \mathrm{B}^{\prime}$. Thus the propagation speed, being equal to the speed of sound minus the flow velocity, is faster 
along $\mathrm{A}^{\prime} \mathrm{B}^{\prime}$ than along $\mathrm{AB}$. As a result, the wave front will rotate in the clockwise direction. This turns the direction of radiation farther to the right, resulting in an almost $80^{\circ}$ direction of propagation (inlet angle).

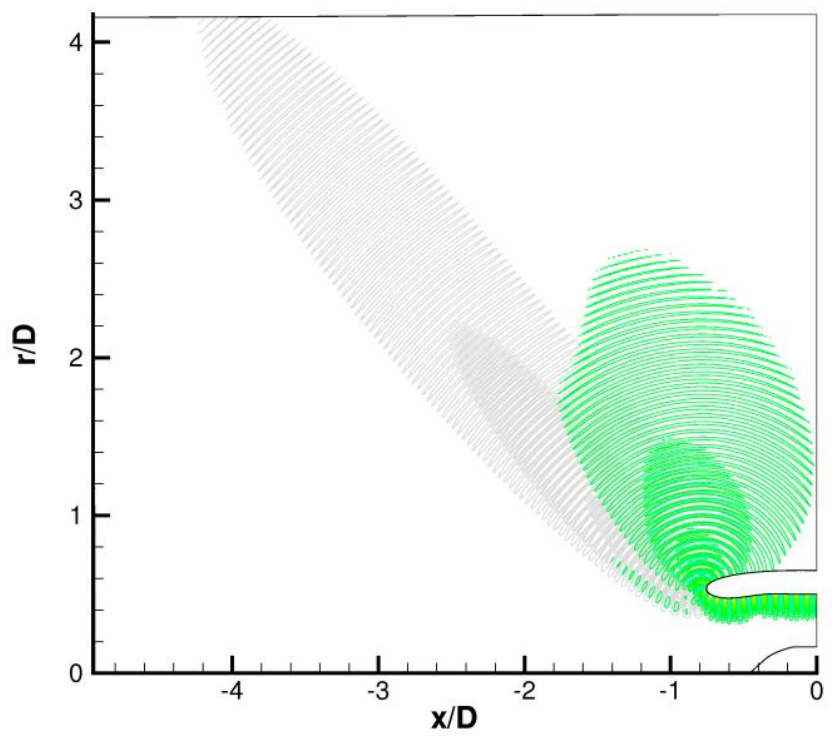

Figure 30. Pressure contours showing the change in the dominant direction of radiation when the fan face Mach number increases from zero (no mean flow, gray) to 0.4 at static condition (green). $m=22, n=1, f=6400 \mathrm{~Hz}$.

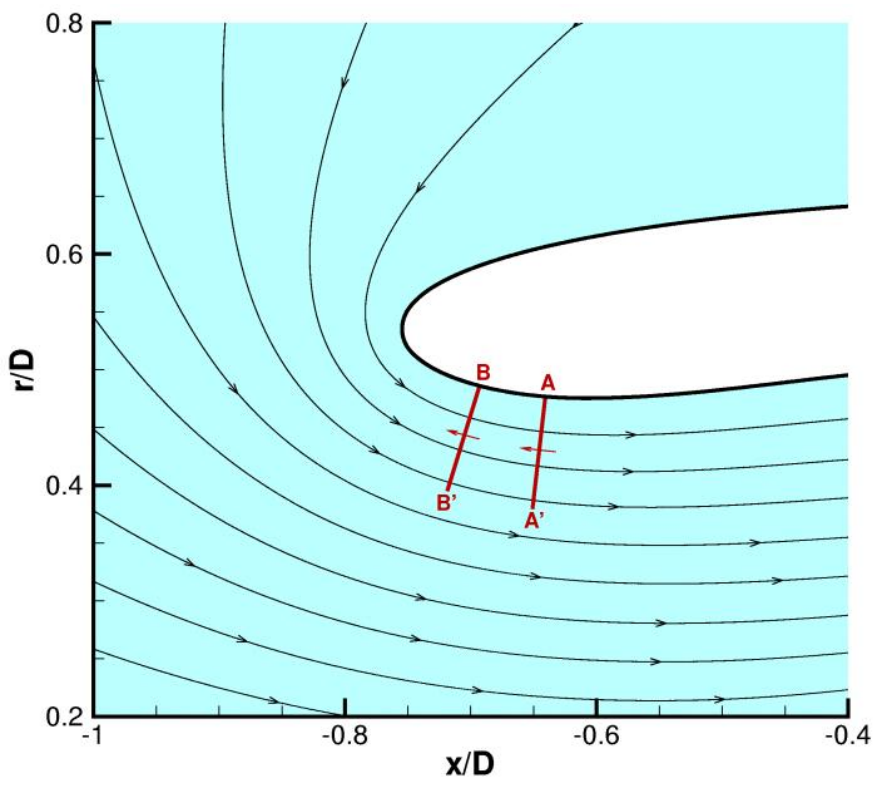

Figure 31. Propagation of wave fronts $\mathrm{AA}^{\prime}$ and $\mathrm{BB}^{\prime}$ along the casing wall at static condition. The wave front rotates in the clockwise direction upon encountering gradients in the mean flow velocity. This is the refraction effect.

For a jet engine in forward flight, the effect of refraction is quite different. Fig. 32 shows the radiation pattern for three mean flow conditions: zero mean flow, static condition, and flight condition. When there is no mean flow, the diffraction effect rotates the dominant direction of radiation to about $45^{\circ}$. In the presence of a mean flow at static condition, refraction further rotates the dominant direction of radiation clockwise to nearly $80^{\circ}$. However, with a forward flight Mach number of 0.2, the refraction effect rotates the dominant direction of radiation by about $10^{\circ}$ counterclockwise off the angle caused by diffraction only. This counterclockwise rotation is not difficult to understand. With forward flight, the mean flow pattern and velocity gradients are shown in Fig. 24. The velocity gradient is almost in opposite direction to that in the static case (see Fig. 22). However, the gradient is milder and occurs over a shorter distance along the casing wall. Again consider the wave fronts $\mathrm{AA}^{\prime}$ and $\mathrm{BB}^{\prime}$ as 
shown in Fig. 33. This time the flow velocity along the casing lip, i.e., $\mathrm{AB}$, is slower than that along $\mathrm{A}^{\prime} \mathrm{B}^{\prime}$. $\mathrm{As}$ a result, the wave front propagates faster along $A B$ than $A^{\prime} B^{\prime}$. This causes a counterclockwise rotation in the direction of wave propagation. This explains the counterclockwise rotation of the dominant direction of radiation for a jet engine in forward flight as observed in Fig. 32. It is to be noted for forward flight condition that the combined effect of diffraction and refraction results in an overall clockwise rotation in the direction of wave propagation. This indicates that the diffraction effect is the more dominant process.

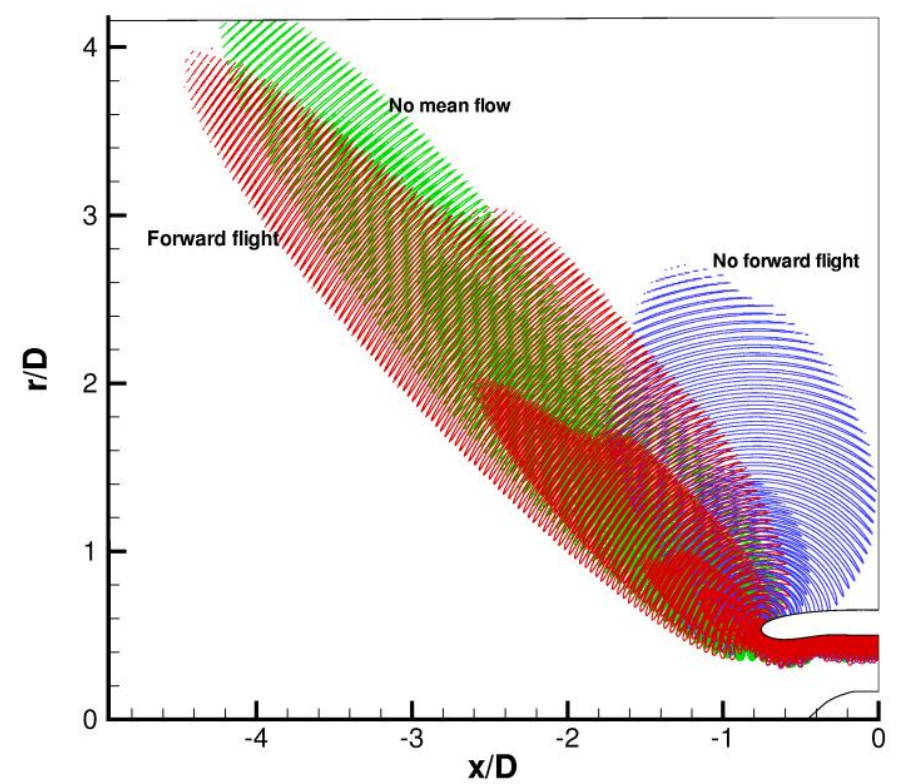

Figure 32. Pressure contours showing the dominant direction of radiation when there is no mean flow (green), when there is a mean flow at static condition (blue; fan face Mach number $=0.4$ ) and when there is a forward flight at Mach 0.2 (red). $m=22, n=1$ and $f=6400 \mathrm{~Hz}$.

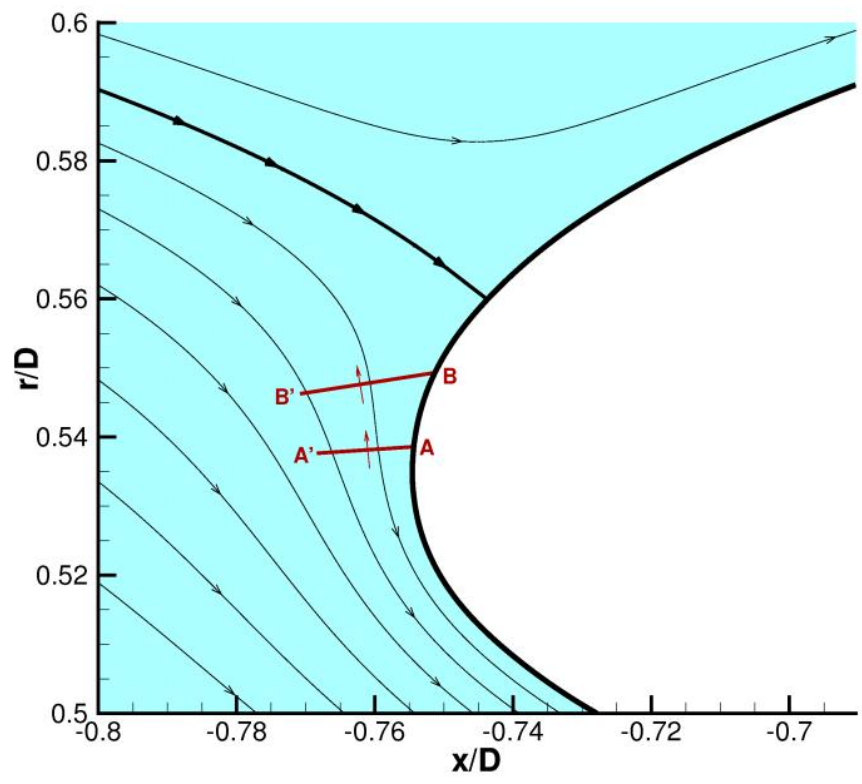

Figure 33. Propagation of wave fronts $\mathrm{AA}^{\prime}$ and $\mathrm{BB}^{\prime}$ when there is a Mach 0.2 forward flight. Here, refraction causes a counterclockwise rotation in propagation.

Just as for diffraction, the effect of refraction is also dependent on the duct mode axial wavelength. Fig. 34 illustrates the propagation of a long wave and a short wave through a region with a localized velocity gradient. Relative to the long wave, the region is small and hence its effect on the wave is minimal. Relative to the short 
wave, the nonuniform flow region is quite large. Thus, the velocity gradient effect is significant. Another way of reaching the same conclusion is by considering the limit of wavelength $\lambda \gg$ length of velocity gradient region. In this limit, the wave effectively does not see the velocity gradient and hence should experience little effect. On the other hand, for waves with wavelengths much shorter than the size of the nonuniform velocity region, one expects the wave to experience the full strength of refraction when traversing the region. Thus the direction of propagation of short waves would be severely turned by refraction, whereas long waves would not be much affected. This observation is summarized symbolically in Fig. 35. In this figure, the case of an engine in static condition is considered separately from the same engine in flight. This is because in static condition, refraction causes the wave propagation direction to rotate in the clockwise direction, while for an engine in forward flight, the refraction effect causes a counterclockwise rotation in the direction of propagation. In both cases, the angle of rotation increases with a decrease in axial wavelength.

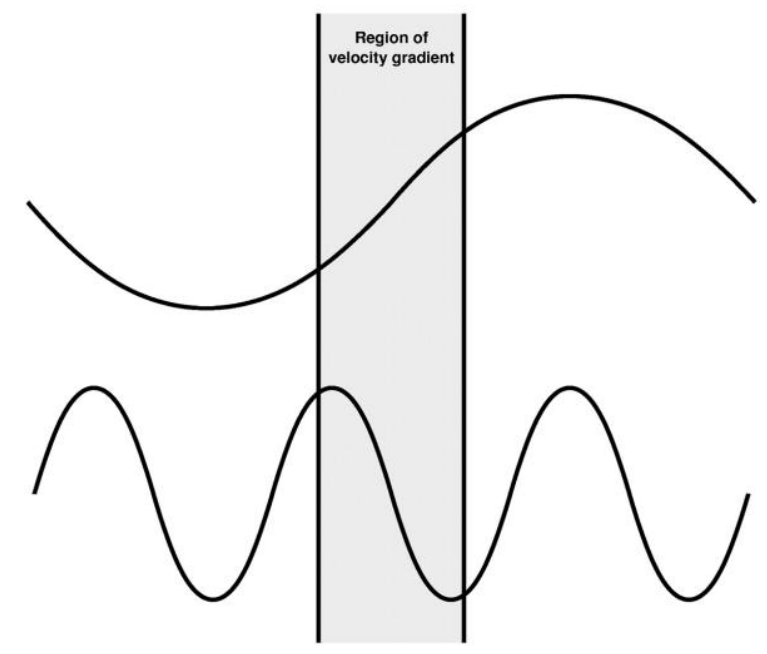

Figure 34. Schematic diagram of the propagation of a long and a short wave through a localized region of velocity gradient.

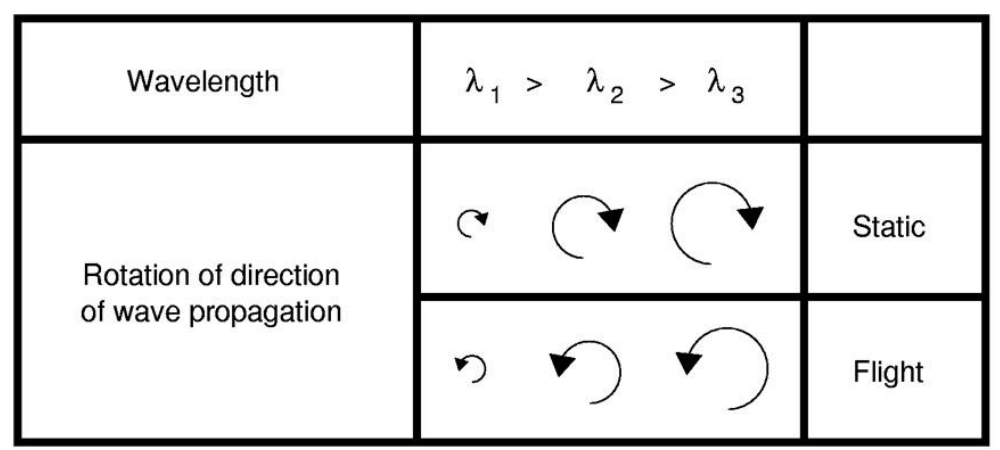

Figure 35. Symbolic representation of dependence of refraction effect on wavelength. Size of arc indicates the magnitude of rotation due to velocity gradient.

\section{Parametric Study of Acoustic Radiation}

We have seen that forward flight has a significant impact on duct mode radiation patterns and directivity. A duct mode is characterized by its azimuthal mode number, radial mode number, and frequency. In this section, the results of a parametric study of the effects of forward flight Mach number, frequency and mode numbers are presented. 


\subsection{Forward flight effect}

The mean flow around an engine inlet changes with forward flight Mach number. Fig. 36 shows the separation streamlines and stagnation point locations for forward flight Mach numbers 0.15, 0.2 and 0.25. This figure shows that as flight Mach number increases, the location of the stagnation point on the casing lip becomes somewhat insensitive to further increase after $\mathrm{M}_{\text {flight }}$ exceeds 0.15. Fig. 37 is similar to Fig. $21 \mathrm{~b}$. It shows the change in flow velocity on the surface of the casing as flight Mach number increases. At zero forward flight Mach number, the velocity on the surface of the casing can speed up to transonic. But for higher flight Mach numbers, the maximum surface velocity is about the same as that at the fan face. This means the refraction effect diminishes at high flight Mach numbers.

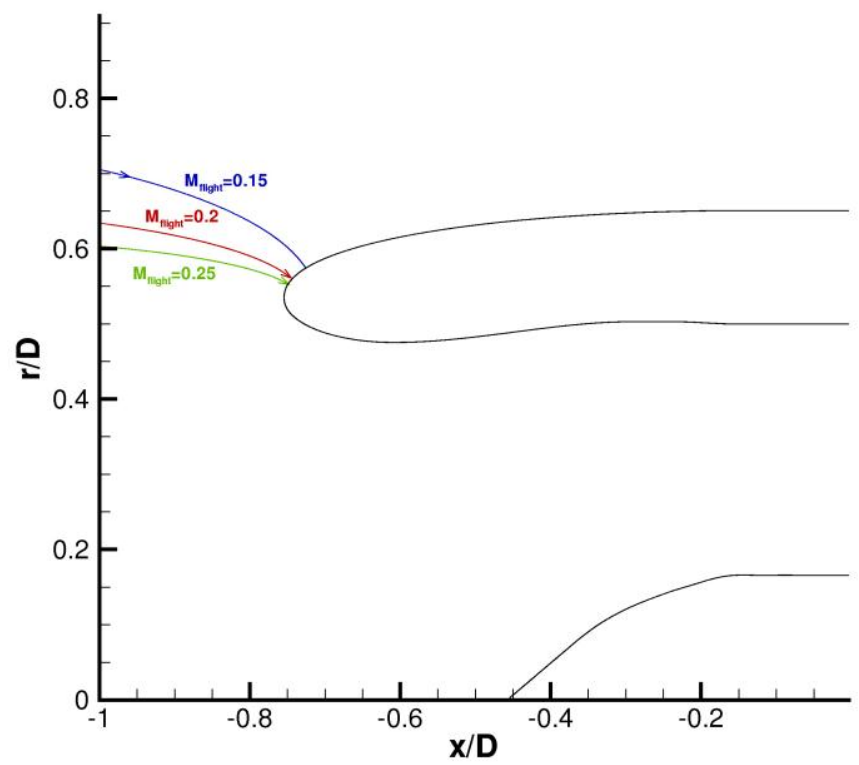

Figure 36. Change in the location of stagnation point as forward flight Mach number increases.

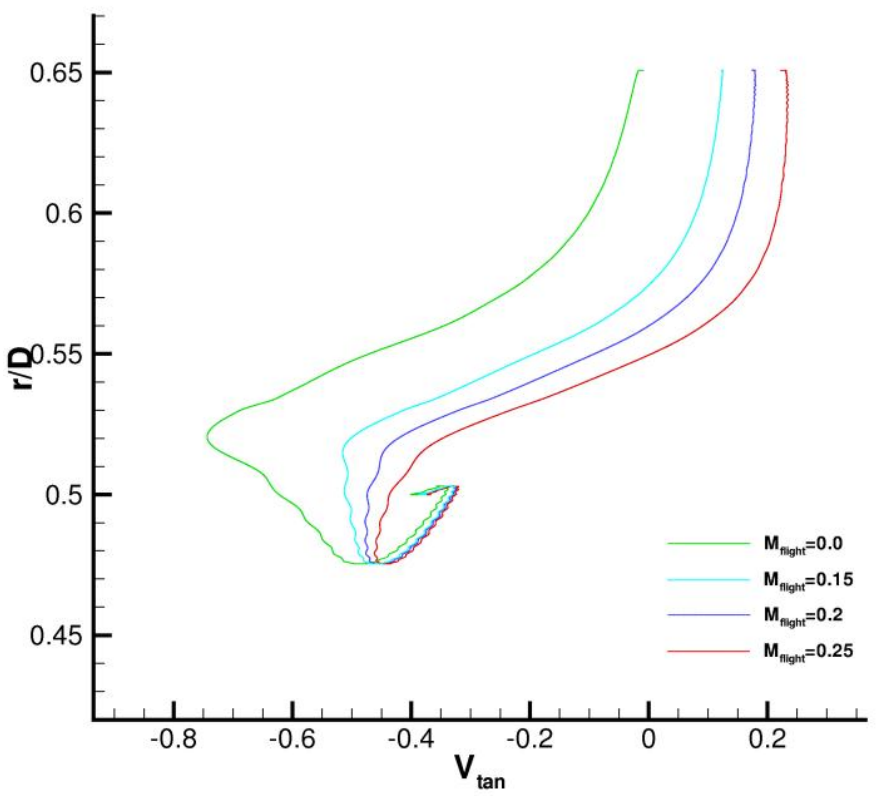

Figure 37. Changes in tangential velocity on engine inlet casing wall as forward flight Mach number increases. 
The effect of forward flight Mach number on directivity is illustrated in Fig. 38. It is clear that there is a saturation effect, namely, beyond flight Mach number 0.15 there is no substantial change in the directivity. A more simplistic way to see this is to look at the direction of maximum radiation. This is shown in Fig. 39. This saturation effect on the impact of increasing flight Mach number occurs because the mean flow undergoes only minor changes in response to such increase beyond 0.15, as was shown in Figs. 36 and 37. Any further refraction effect is minimal, leading to little change in directivity.

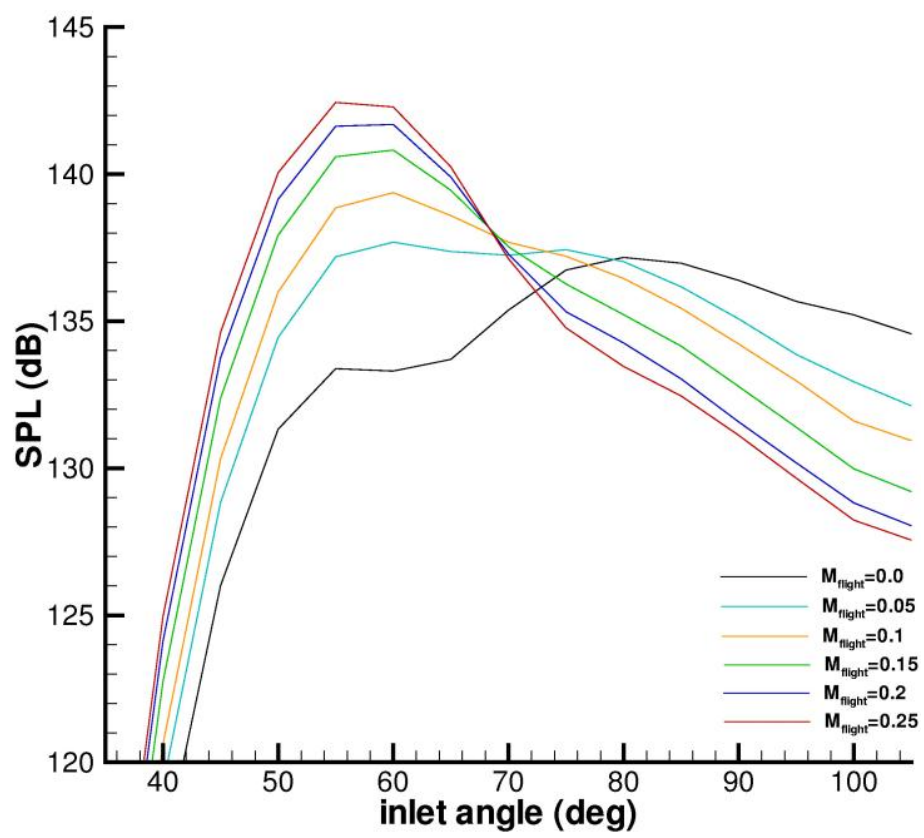

Figure 38. Directivity of the radiation of duct mode $m=22, n=1, f=5600 \mathrm{~Hz}$ (fan face Mach number 0.4 ) at varying forward flight Mach numbers.

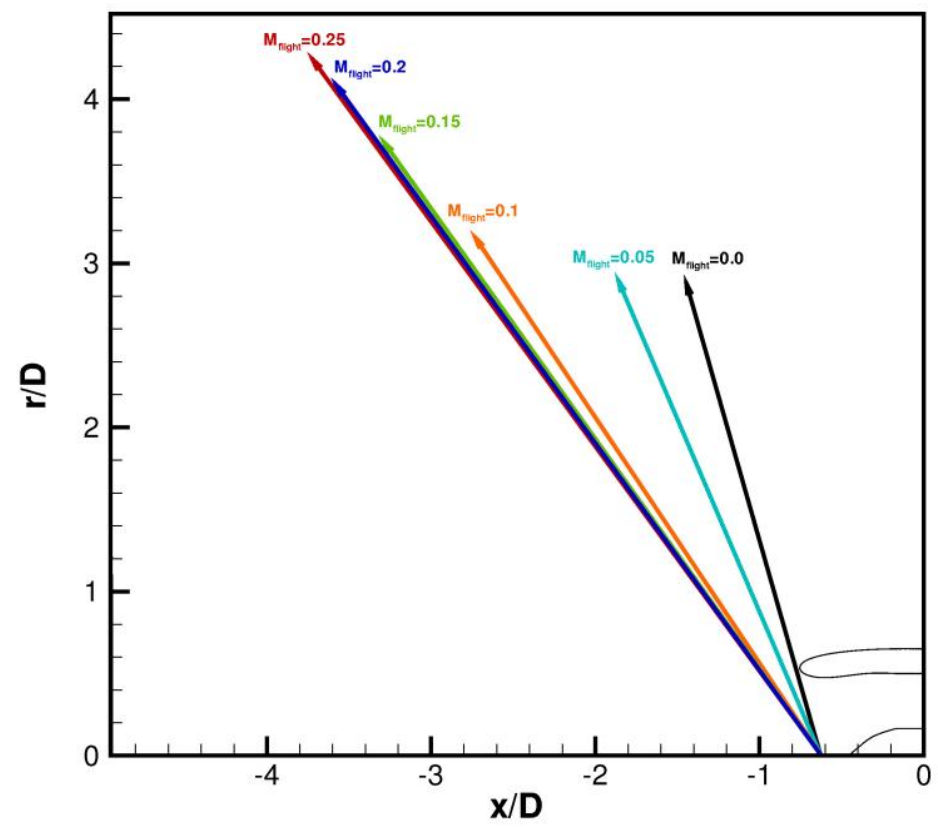

Figure 39. Direction of maximum sound radiation at varying forward flight Mach numbers. Duct mode with $m=22, n=1, f=5600 \mathrm{~Hz}$, and fan face Mach number 0.4. 


\subsection{Frequency variation}

The axial wavelength of a duct mode changes with frequency. Fig. 13 shows the dependence of the duct mode axial wavelength on frequency for the case $M_{\text {fan }}=0.4$ at static condition. As discussed before, both diffraction and refraction effects are axial-wavelength dependent. To investigate the dependence of the directivity of radiation on frequency, we will consider the case $M_{\text {fan }}=0.4, m=22$ and $n=1$. Fig. 40 shows the pressure contour patterns at static condition, i.e., $M_{\text {flight }}=0$, when the duct mode frequency varies from 4.5 to $6.4 \mathrm{kHz}$. It is clear from this figure that the pattern changes only slightly with frequency. Now, let us consider the case at forward flight Mach number 0.2. The pressure contour patterns are displayed in Fig. 41. At $M_{\text {fight }}=0.2$, the dominant direction of radiation is a strong function of frequency. Higher-frequency duct modes radiate at lower inlet angles. Thus, there is a significant difference in the radiation pattern with and without forward flight. This phenomenon may also be observed by considering directivity. The directivities are shown in Fig. 42 for $M_{\text {flight }}=0$ and Fig. 43 for $M_{\text {flight }}=0.2$. Again, there is little frequency dependence at the static condition, but a significant dependence exists when there is forward flight.

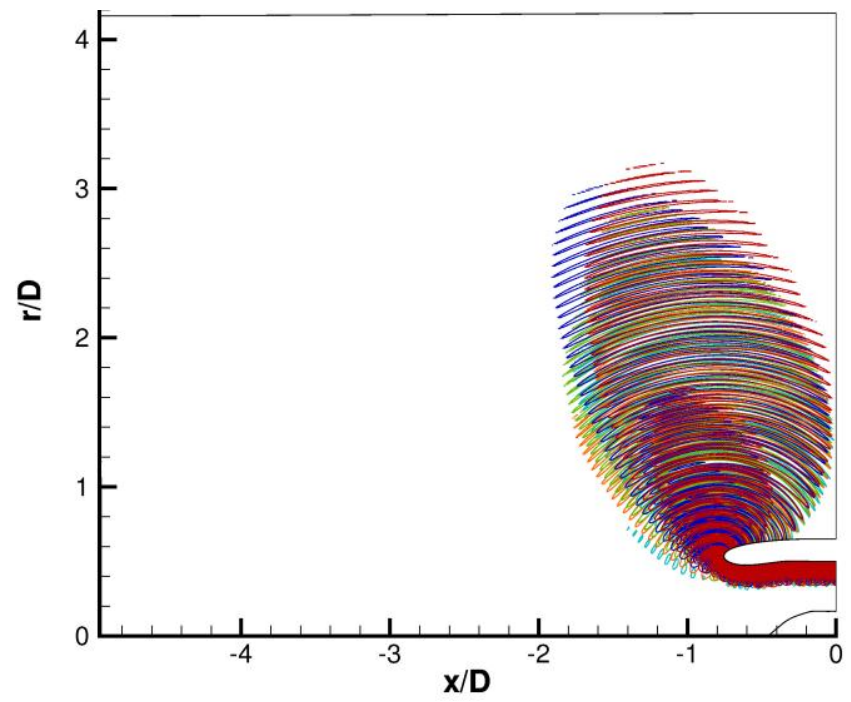

Figure 40. Pressure contour pattern of duct mode radiation. $M_{\text {fan }}=0.4, M_{\text {flight }}=0, m=22, n=1$, with frequencies varying over $f=4.5,4.8,5.4,5.6$ and $6.4 \mathrm{kHz}$.

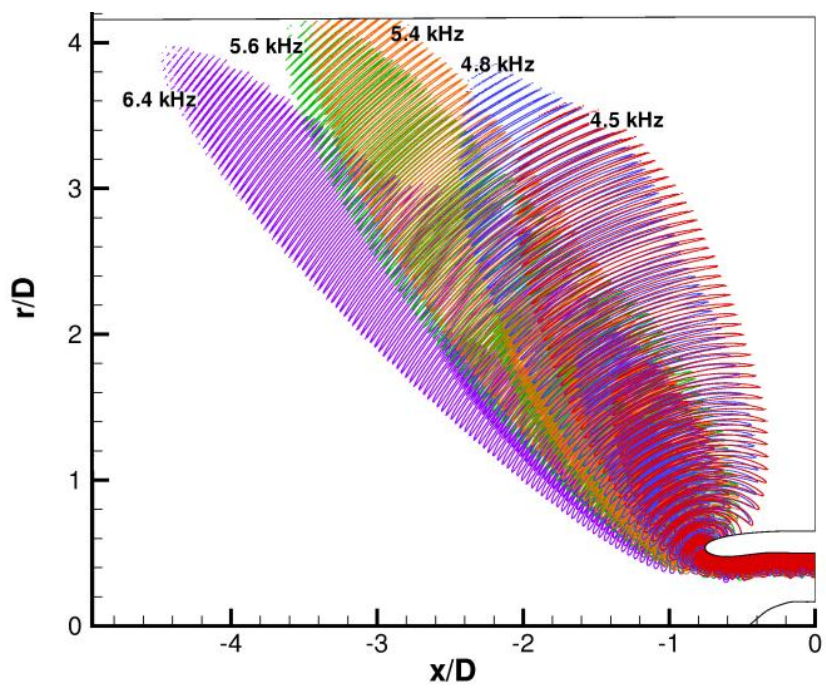

Figure 41. Pressure contour pattern of duct mode radiation. $M_{\text {fan }}=0.4, M_{\text {flight }}=0.2, m=22, n=1$, with frequencies varying over $f=4.5,4.8,5.4,5.6$ and $6.4 \mathrm{kHz}$. 


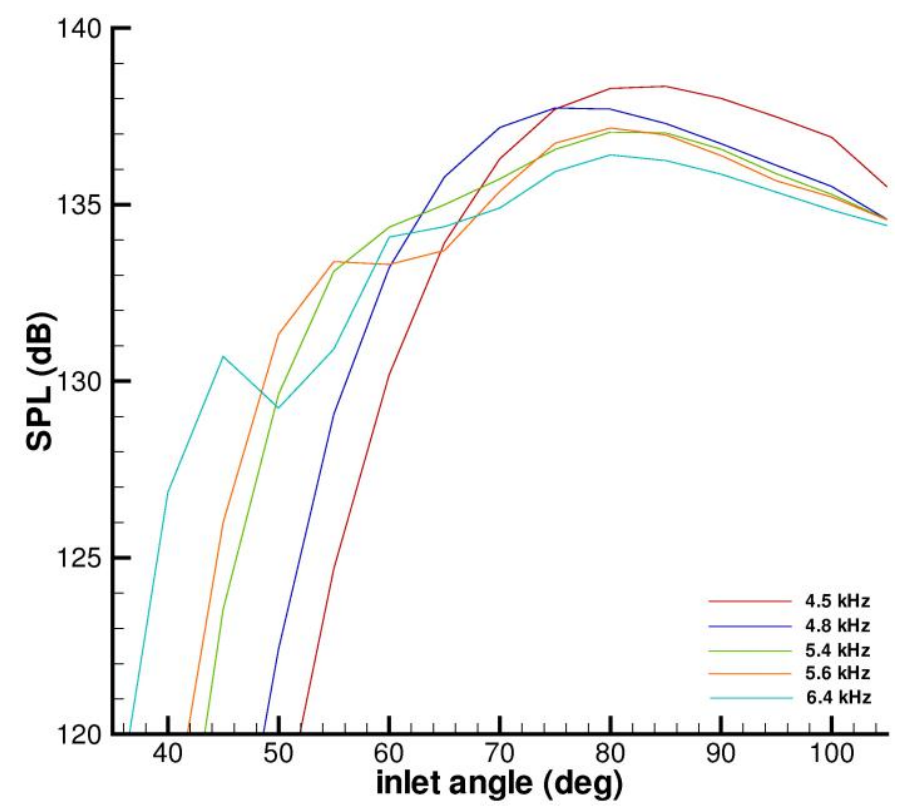

Figure 42. Directivities of duct mode radiation. $M_{\text {fan }}=0.4, M_{\text {flight }}=0, m=22, n=1$.

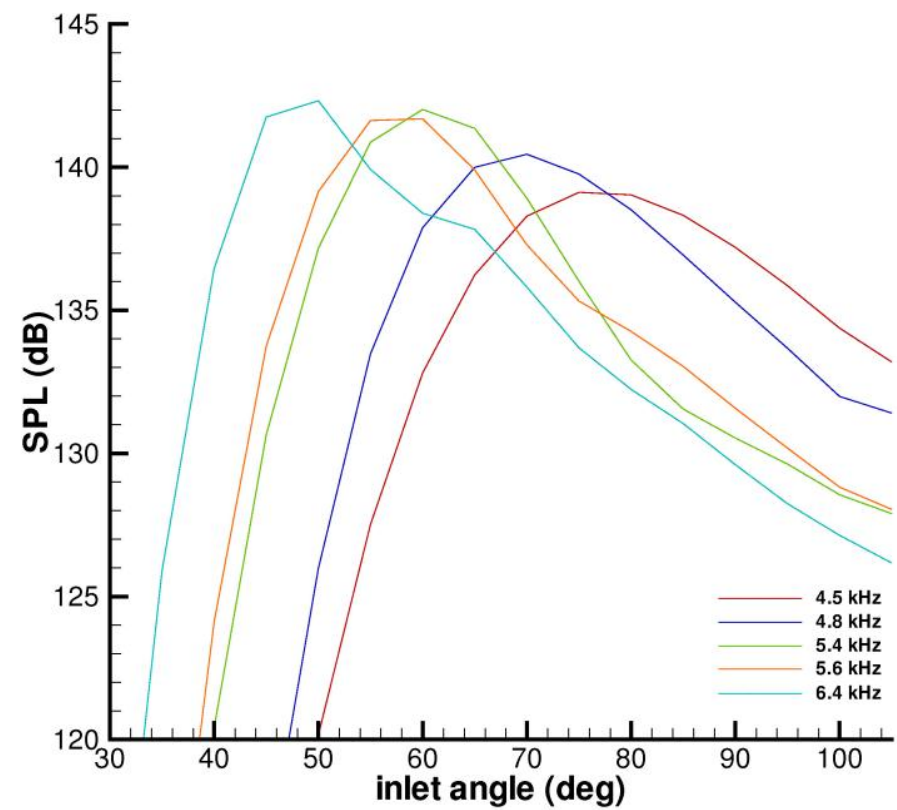

Figure 43. Directivities of duct mode radiation. $M_{\text {fan }}=0.4, M_{\text {flight }}=0.2, m=22, n=1$.

This frequency-dependence phenomenon can be explained by recalling the dependence of the effects of diffraction and refraction on the axial wavelength of duct modes, discussed in Sections 5.1 and 5.2. It is to be noted that duct mode axial wavelengths are shorter at higher frequencies. Thus, the diffraction effect has less impact on high-frequency duct modes. But they are subjected to a stronger refraction effect. On the other hand, for lowfrequency duct modes with longer wavelengths, the diffraction effect is larger and refraction effect smaller. At static condition, both effects cause clockwise rotation of the direction of radiation. As a result, the combined effects lead to nearly the same amount of total rotation in the direction of radiation, regardless of wave frequency, as in Fig. 40. This is shown symbolically in the first column labeled 'static' in Fig. 44. When the engine is in forward flight, the total effect is different. This is because the refraction effect now turns the direction of radiation counterclockwise, opposite to the effect of diffraction. For low-frequency long waves, clockwise turning by diffraction is larger, and the counterclockwise turning by refraction is smaller. For high-frequency short waves, the clockwise turning by 
diffraction is small, while the counterclockwise turning by refraction is relatively large. The combined result is that low-frequency long waves radiate at a larger inlet angle, while high-frequency short waves radiate at a lower inlet angle, as in Fig. 41. This is illustrated symbolically in the second column of Fig. 44.

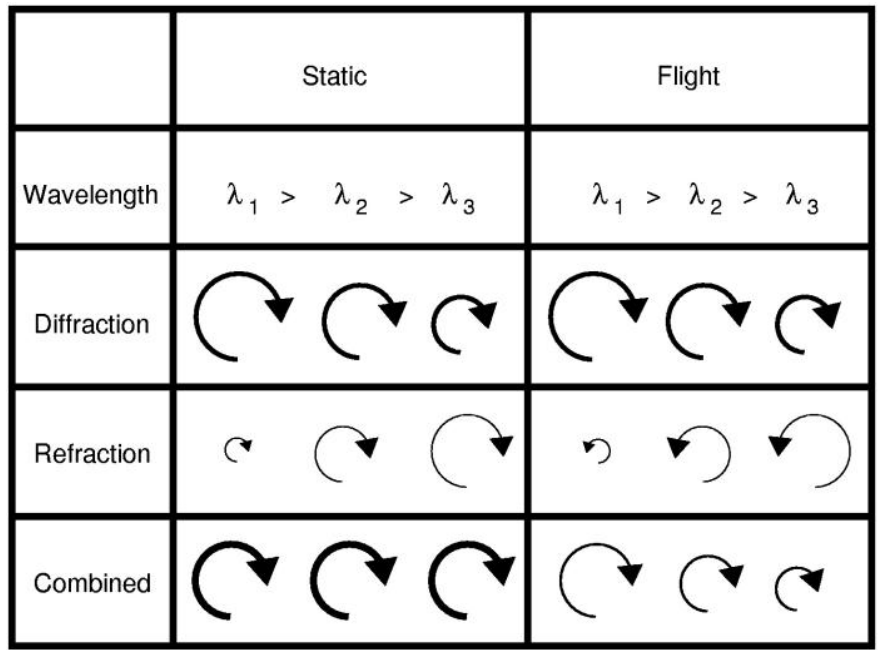

Figure 44. Symbolic representation of the independent and combined effects of diffraction and refraction with and without forward flight. Size and thickness of circular arc indicate the relative magnitude of the turning angle.

\subsection{Azimuthal mode number variation}

At a fixed frequency and radial mode number, the axial wavelength of an upstream propagating duct mode decreases with a decrease in azimuthal mode number. Fig. 14 shows the case of $M_{\text {fan }}=0.4, M_{\text {flight }}=0, n=1, f=6400$ $\mathrm{Hz}$ for the SDT engine inlet. Thus, it is expected that a variation in azimuthal mode order would have a similar effect on the radiation directivity as the effect of frequency variation. Fig. 45 shows the pressure contour patterns of radiation for the cases with $M_{\mathrm{fan}}=0.4, M_{\mathrm{flight}}=0, n=1, f=6400 \mathrm{~Hz}$ and $m=18,22,26$ and 30 . The results suggest that at static condition, there is little change in the dominant direction of radiation. Fig. 46 is the same as Fig. 45, except with $M_{\text {flight }}$ equal to 0.2. Now there is a spread of the dominant direction of radiation for varying values of $m$. The observed azimuthal mode number effect is similar to that due to frequency variation. In fact, just like frequency, the observed phenomenon is caused by the dependence of diffraction and refraction effects on wavelength. Lowering the azimuthal mode number leads to a reduction in axial wave number. This is the same as an increase in wave frequency. So the explanation for frequency dependence also applies to azimuthal mode number dependence.

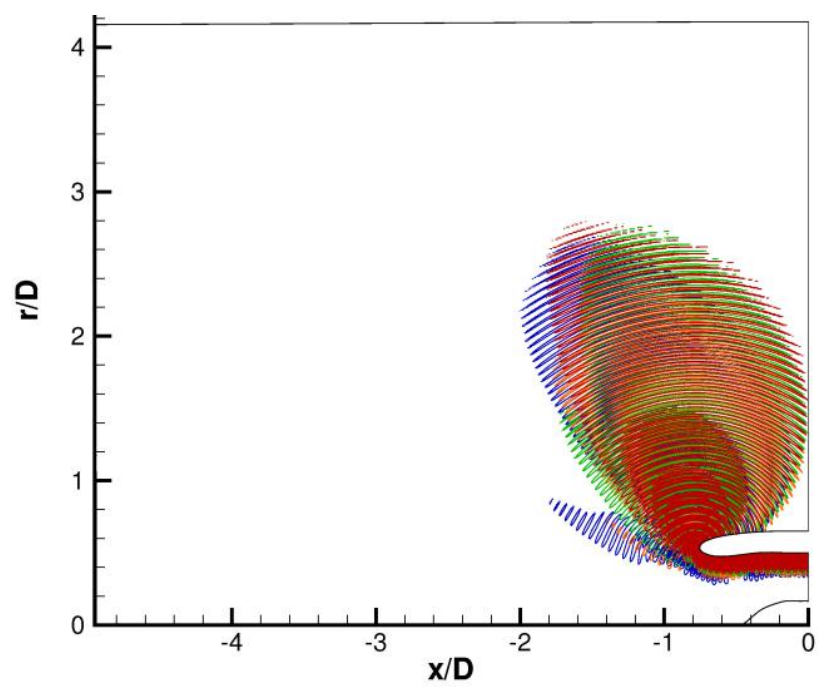

Figure 45. Pressure contour patterns associated with duct mode radiation from an SDT engine inlet.

$$
M_{\text {fan }}=0.4, M_{\text {flight }}=0, n=1, f=6400 \mathrm{~Hz} \text {, and } m=18,22,26,30 \text {. }
$$




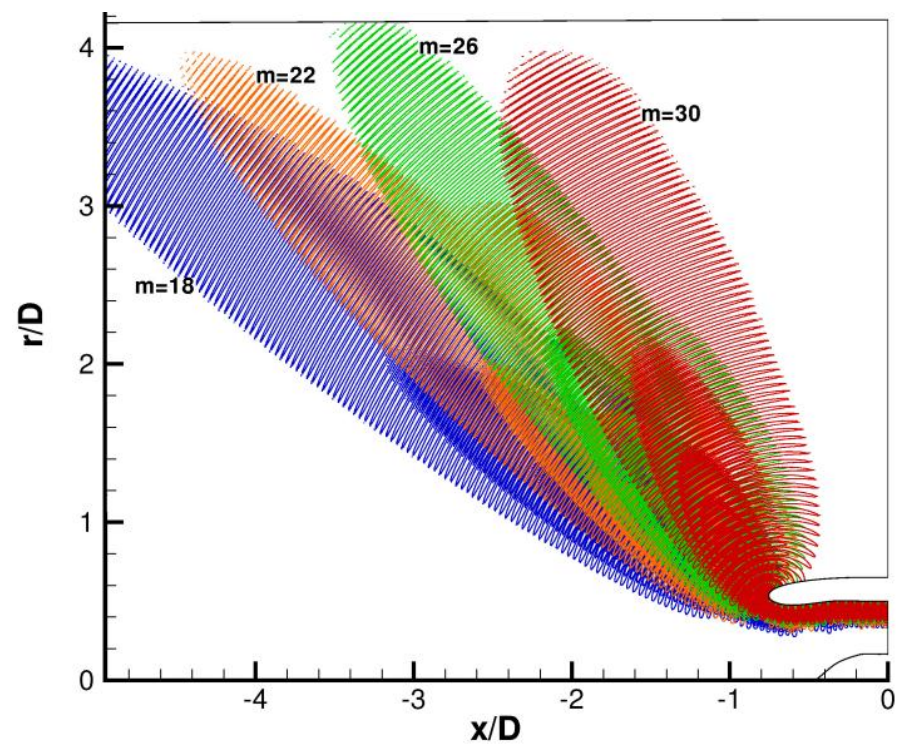

Figure 46. Pressure contour patterns associated with duct mode radiation from an SDT engine inlet.

$$
M_{\text {fan }}=0.4, M_{\text {flight }}=0.2, n=1, f=6400 \mathrm{~Hz} \text {, and } m=18,22,26,30 .
$$

\subsection{High-order radial modes}

Radial mode number $n$ (as defined in this investigation) is the sum of the total number of maxima and minima of the duct mode eigenfunction. Because a wave oscillates in time, a duct mode with radial mode number $n$ will have a wave field with $n$ rows of pressure maxima and minima. The high and low pressure regions alternate in a staggered pattern (see Fig. 47). Fig. 47 shows the pressure field contour pattern of the $n=2$ duct mode at $M_{\text {fan }}=0.0$, $M_{\text {flight }}=0.0, m=22, f=6400 \mathrm{~Hz}$. Notice there are two rows $(n=2)$ of alternating high and low pressure regions. As diffraction causes the wave field to follow the turn of the casing lip, it is seen that the duct mode splits into two beams. Apparently, the cause of this beam splitting is that the row of pressure maxima and minima adjacent to the wall is influenced more by diffraction. Thus, it turns clockwise more than the second row of pressure maxima and minima farther from the wall.

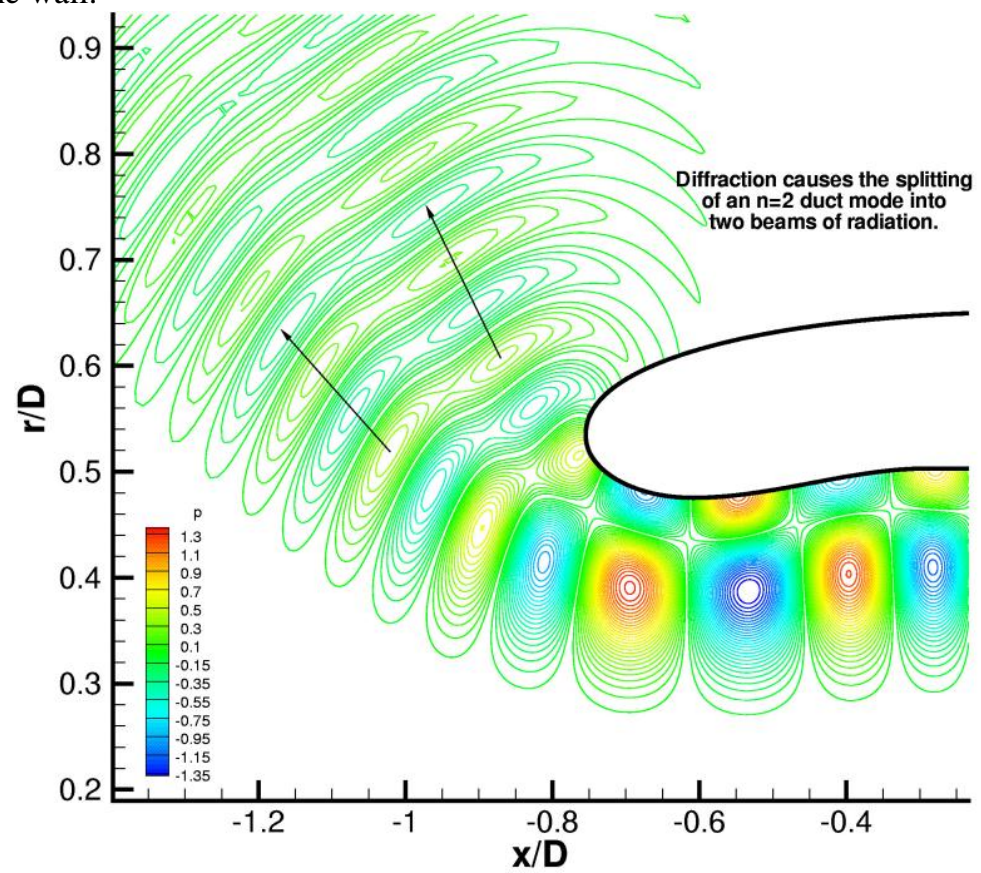

Figure 47. Near field pressure contours showing the splitting of an $n=2$ duct mode into two beams.

$$
M_{\text {fan }}=0.0, M_{\text {flight }}=0.0, m=22, n=2, f=6400 \mathrm{~Hz}
$$


This beam-splitting phenomenon for the second radial mode is affected by forward flight Mach number. Figs. $48 \mathrm{a}-48 \mathrm{c}$ show the change in the pressure contour pattern for the case $M_{\mathrm{fan}}=0.4, m=22, f=5600 \mathrm{~Hz}$, as flight Mach number increases. Fig. 48 a corresponds to $M_{\text {flight }}=0.0$; Fig. $48 \mathrm{~b}$ is for $M_{\text {flight }}=0.15$; and Fig. $48 \mathrm{c}$ for $M_{\text {flight }}=$ 0.25 . By comparing these figures, it should be obvious that forward flight has a significant influence on the radiation pattern and directivity. Fig. 49 is a directivity plot for $M_{\text {flight }}=0.05,0.1,0.15,0.2$ and 0.25 . There are basically two beams of radiation. As flight Mach number increases, there is a gradual changeover in the dominancy of the two beams. At low flight Mach numbers, the beam radiated to the lower inlet angle is the dominant beam. As $M_{\mathrm{flight}}$ increases, a gradual shift in dominancy takes place. By $M_{\text {flight }}=0.15$, the shift is nearly complete. Beyond 0.15 , the beam that radiates to the higher inlet angle is the dominant beam.

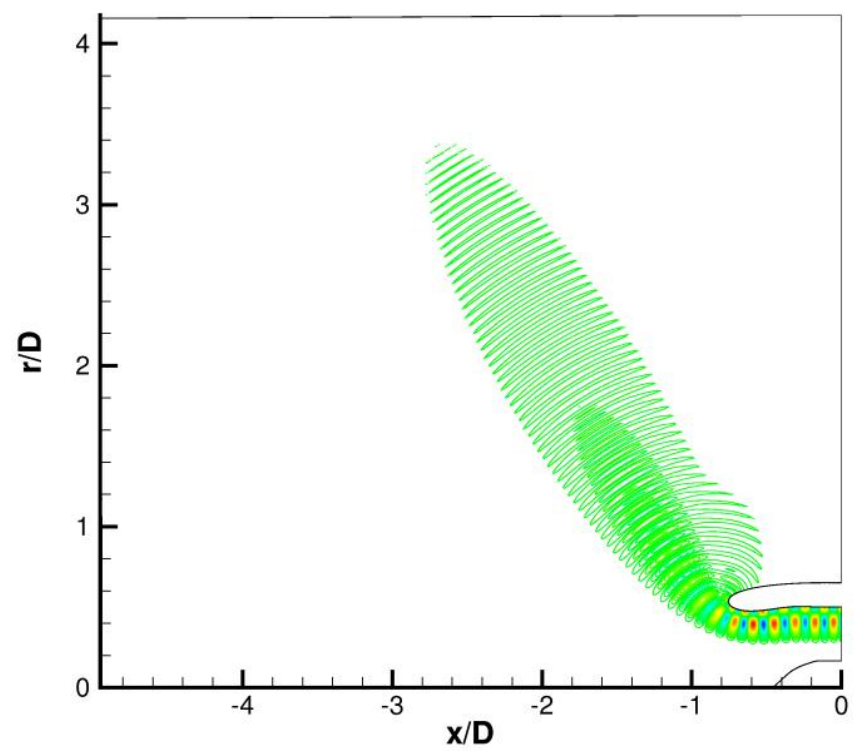

Figure 48a. Radiation pattern from an $n=2$ duct mode with $m=22, M_{\text {fan }}=0.4, M_{\text {flight }}=0.0, f=5600 \mathrm{~Hz}$.

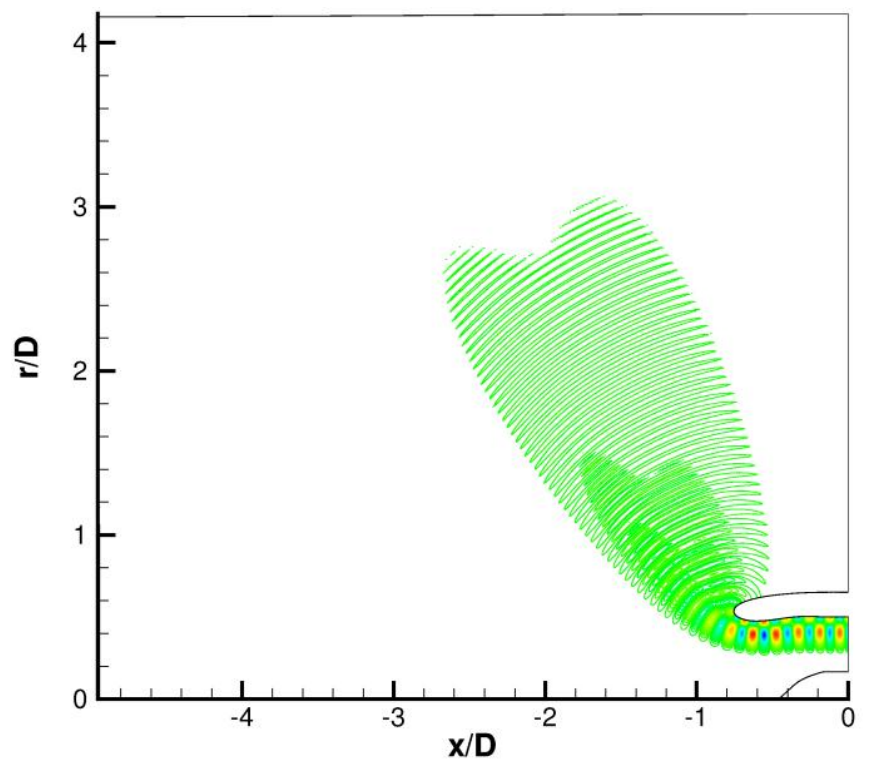

Figure 48b. Radiation pattern from an $n=2$ duct mode with $m=22, M_{\text {fan }}=0.4, M_{\text {flight }}=0.15, f=5600 \mathrm{~Hz}$. 


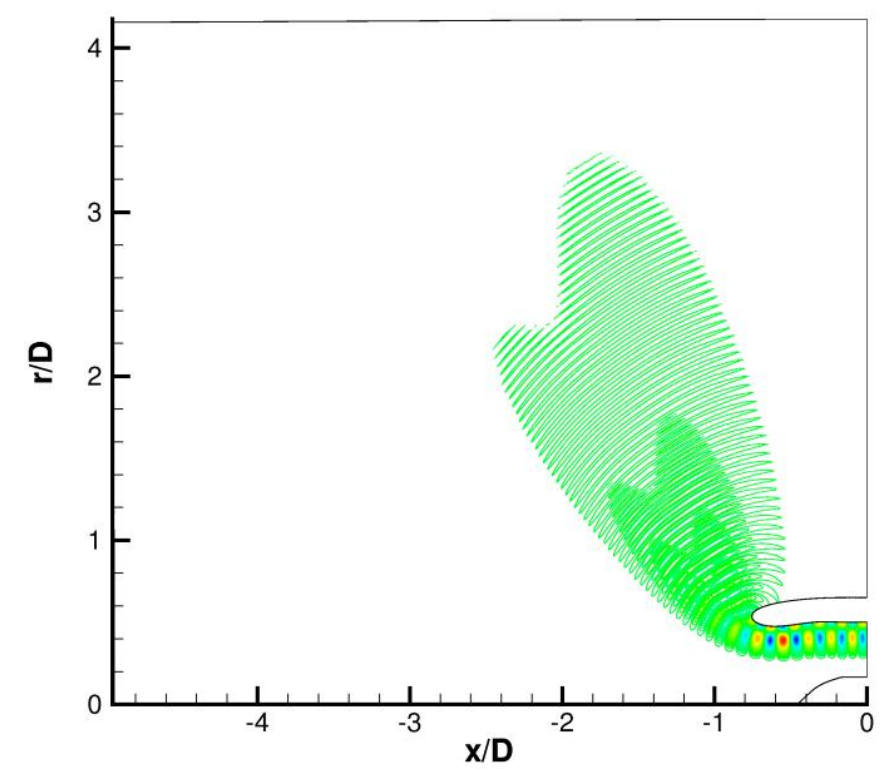

Figure 48c. Radiation pattern from an $n=2$ duct mode with $m=22, M_{\text {fan }}=0.4, M_{\text {flight }}=0.25, f=5600 \mathrm{~Hz}$.

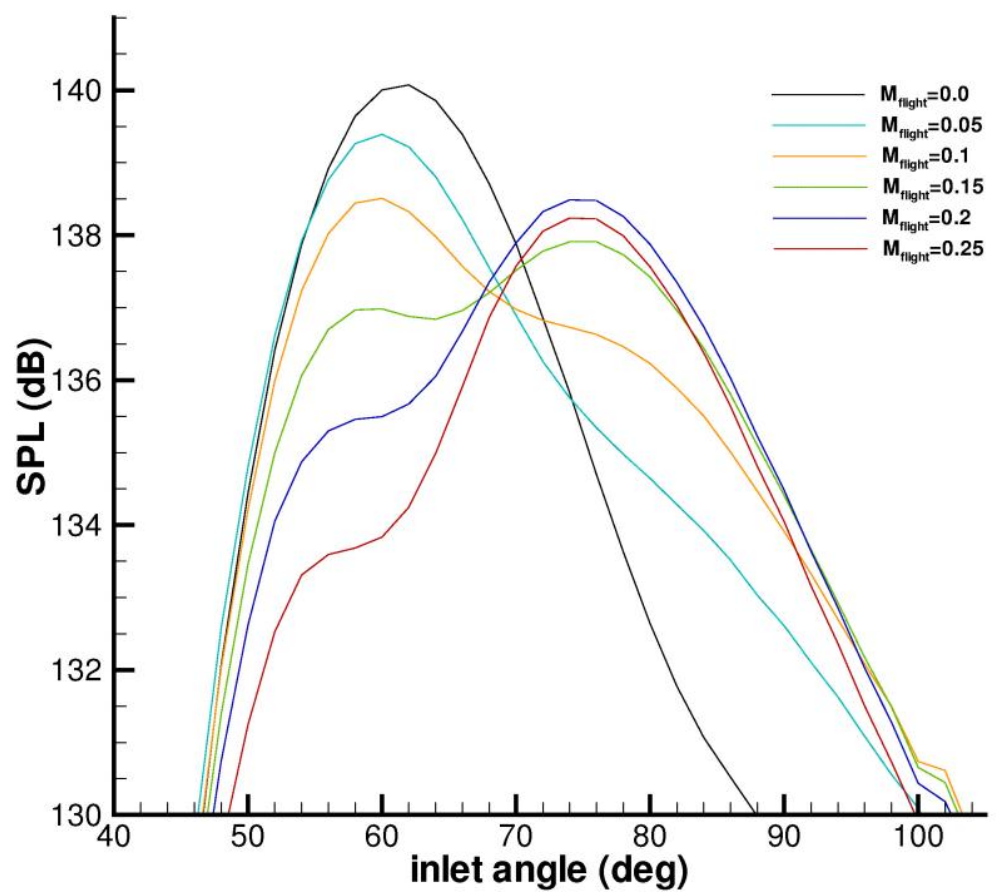

Figure 49. Directivity of sound in the far field radiated by an $n=2$ duct mode with $m=22, M_{\text {fan }}=0.4, f=5600 \mathrm{~Hz}$.

Fig. 50 shows the results of taking an even higher radial mode number: $M_{\text {fan }}=0.4, M_{\text {flight }}=0.2, m=22, n=$ $4, f=6800 \mathrm{~Hz}$. Upon radiating out of the engine inlet, the four rows of high and low pressure regions merge into two beams. It is to be noted that the radial mode number $n$ is meaningful inside the duct and in the part of the inlet close to the fan face. Once the sound waves exit the inlet, the radial mode number is no longer meaningful. Therefore, at the engine inlet, there is a region in which a high-order radial mode would undergo a transformation whereby the multi-layer pressure field structure combines into a single sinusoidal spatial structure typical of far field propagating acoustic waves. In addition, inside an engine inlet, the sound field of a duct mode has an axial wavelength determined by frequency, azimuthal mode number, mean flow Mach number and the diameter of the duct. But once the sound field exits the inlet, the wavelength of the freely propagating acoustic wave depends solely on its frequency. Therefore, there is also an adjustment of wavelength in the transition region. This wavelength transition 
also applies to duct modes with $n=1$ radial mode number. Fig. 51 shows the transition for an $n=4$ duct mode in the engine inlet.

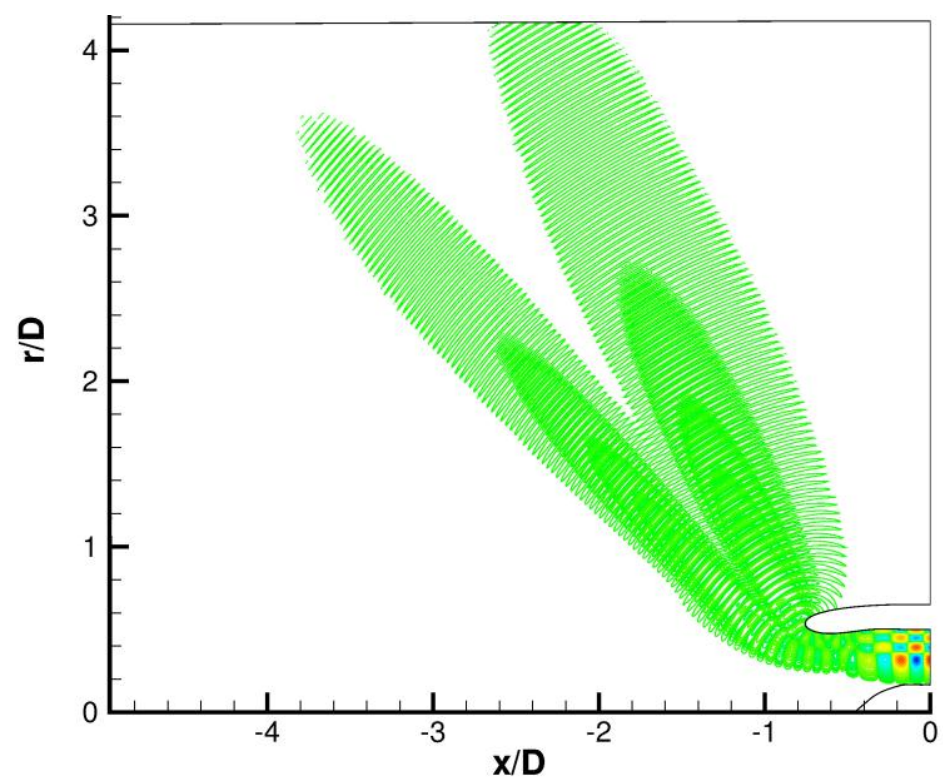

Figure 50. Radiation pattern for an $n=4$ duct mode with $m=22, M_{\mathrm{fan}}=0.4, M_{\mathrm{flight}}=0.2, f=6800 \mathrm{~Hz}$. The four rows of high and low pressure fluctuations near the fan face eventually radiate out of the inlet as two beams.

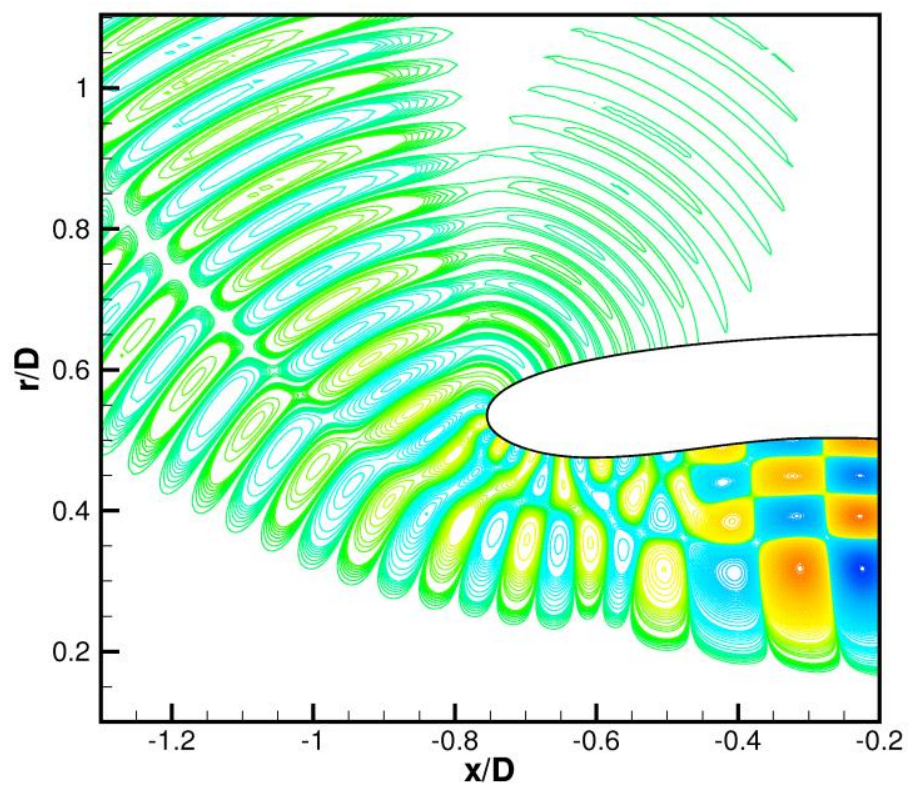

Figure 51. Pressure contours showing the transition region at the inlet of an engine for an $n=4$ duct mode. $m=22, M_{\text {fan }}=0.4, M_{\text {flight }}=0.0, f=6800 \mathrm{~Hz}$.

\section{Summary and Conclusion}

Numerical simulation of duct mode radiation from a jet engine inlet is carried out. An advanced CAA time marching algorithm and high-quality numerical boundary conditions are used. The computational code is validated by comparing predicted directivities for the JT15D engine with experimental measurements. 
Computed results are used to highlight the important physical processes that affect the sound radiation pattern and directivity. It is demonstrated that the phenomena of diffraction and refraction play a key role in determining the ultimate direction of radiation. Diffraction is the natural tendency for a duct mode to follow a curved solid surface as it propagates. Refraction is the bending of the direction of sound propagation by a mean flow velocity gradient. It is shown that the mean flow velocity distribution around an engine inlet in forward flight differs markedly from that in static operating condition. For this reason, there is a substantial change in the duct mode radiation pattern and directivity when switching from static condition to forward flight condition. However, as flight Mach number increases beyond 0.15 , there are only minor changes in the mean flow. In other words, the forward flight effect exhibits a saturation phenomenon. That is, further increase in forward flight Mach number above 0.15 would bring about little change in the duct mode radiation directivity.

In this investigation, a parametric study of the effect of frequency and azimuthal mode number on inlet acoustic radiation has been carried out. Both frequency and azimuthal mode number affect the axial wavelength of a duct mode. It is found that a change in axial wavelength affects the level of influence of diffraction and refraction. Refraction exerts a larger impact on short waves than on long waves. Thus duct modes with higher frequencies or lower azimuthal mode numbers are more affected by refraction. On the other hand, diffraction has a larger effect on duct modes with long axial wavelengths. Because of these differences in axial-wavelength dependence, when the frequency or azimuthal mode number changes, the combined effect of diffraction and refraction causes most duct modes at static condition to radiate at a relatively consistent angle in the sideline direction (approximately $80^{\circ}$ inlet angle) and duct modes at forward flight condition to radiate at a range of angles in the forward direction.

A duct mode with a radial mode number $n$ has $n$ rows of alternating high and low pressure regions. At the casing lip region, because different rows are at different distances from the wall, they are subjected to different degrees of diffraction and refraction. This results in the splitting of the radiation pattern into multiple beams. The results of a brief study of this effect on duct modes with high-order radial mode numbers is reported.

It seems worthwhile to emphasize, based on the numerical simulation results of the present investigation, that the duct mode radiation pattern from a jet engine inlet in forward flight is quite different from that in static condition. This suggests, for community noise prediction purposes, there is no simple scaling formula that can convert the radiation pattern from the static condition to the flight condition. It is also recognized that there is no simple way to create a stagnation point, a distinct characteristic of forward flight, on the casing lip of an engine in a static test condition. Therefore, static engine test data might not be of use for engines in flight. Hence, their usefulness is very limited.

Before the advent of fast computers, sound radiation from engine inlets was investigated using a somewhat idealized model. The work of Homicz and $\mathrm{Lordi}^{11}$, $\mathrm{Candel}^{43}$, Wright ${ }^{44}$ and others adopted a zero-thickness cylindrical inlet model. One main advantage of such a model is that the duct mode radiation problem can be solved exactly by the Wiener-Hopf technique. These early works were later summarized and extended by Rice, Heidmann and Sofrin ${ }^{45}$ to form a prediction formula for the direction of peak noise radiation. The most general formula of Rice et al. was designed to be applicable to inlet radiation at static as well as at flight condition. In their paper, they pointed out that in deriving their formula, only the mean flow convection effect was included. The refraction effect was entirely neglected. In a real engine, the engine casing has a finite thickness. As was discussed earlier in Section 5 , this thickness is very important to the processes of diffraction and refraction. Setting this thickness to zero may lead to severe prediction error. 


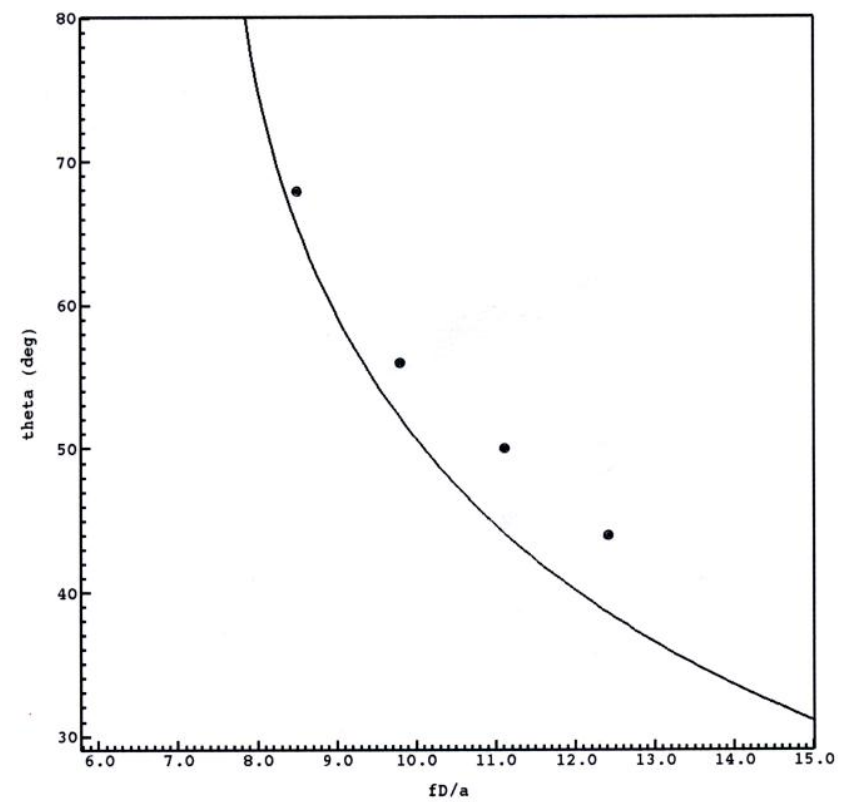

Figure 52. Peak direction of radiation for a duct mode with $m=22, n=1$ as a function of dimensionless frequency with no mean flow. Rice et al. theory ${ }^{45}$ (zero-thickness cylindrical inlet), numerical simulation (SDT engine inlet).

As an illustration of the influence of inlet casing thickness on the effect of diffraction, a comparison between the computed peak direction of radiation from an SDT engine inlet using numerical simulation and that from a zero-thickness cylindrical inlet of the same diameter using the Rice et al. theory ${ }^{45}$ (in the absence mean flow) is made. Fig. 52 shows a comparison of the computed peak directions of radiation for a duct mode with $m=22$ and $n=1$ as a function of frequency. The relevant parameter, important to the diffraction process, is the ratio of casing thickness to the axial wavelength of the duct mode. For the SDT inlet, this ratio is finite; for the zero-thickness cylindrical inlet, this ratio is zero. At low frequencies, the duct mode axial wavelength is long, so the thickness-towavelength ratio is small. In this case, one would expect the two predictions to be fairly close. This is confirmed in Fig. 52. As frequency increases, this ratio becomes larger and larger for the SDT inlet. It follows that the difference between the two computed peak directions of radiation becomes larger and larger. This is evident in Fig. 52.

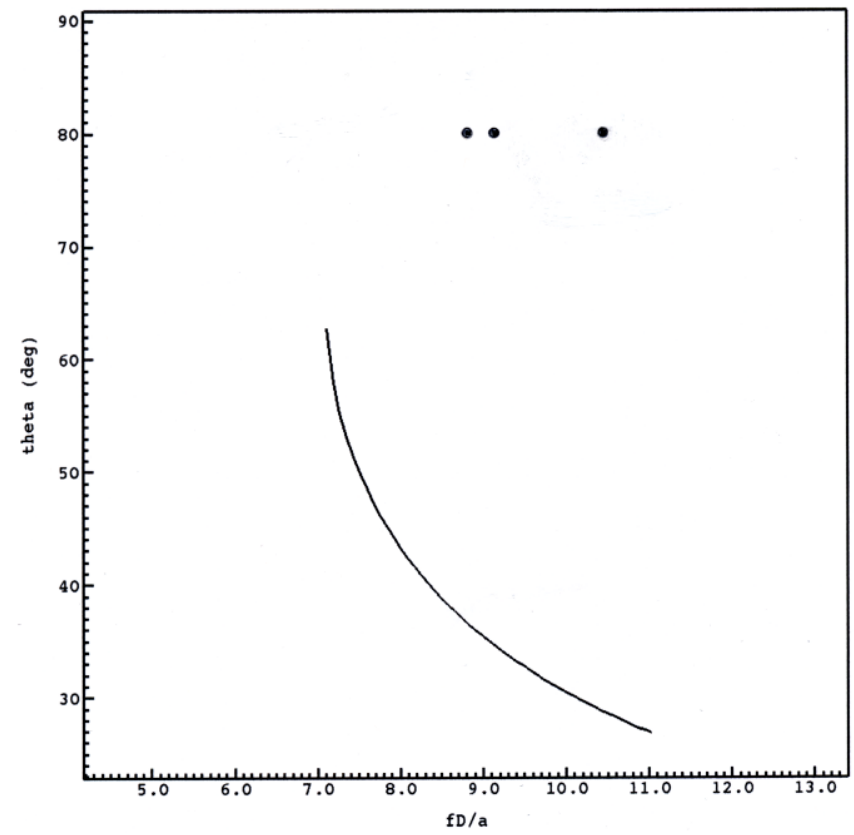


Figure 53. Peak direction of radiation for a duct mode with $m=22, n=1$ as a function of dimensionless frequency. Static engine test with $M_{\mathrm{fan}}=0.4$. $\longrightarrow$ Rice et al theory ${ }^{45}$ (zero-thickness cylindrical inlet), $\bullet$ numerical simulation (SDT engine inlet).

As an illustration of the importance of including mean flow refraction in the prediction of duct mode radiation, a comparison is made between the result of using the classical theory of Rice et al. ${ }^{45}$ and that obtained by numerical simulation for the SDT engine inlet at static test condition. Fig. 53 shows the peak directions of radiation for a duct mode with $m=22$ and $n=1$ at different dimensionless frequencies. It is clear that there are huge differences between the two predictions. Based on this result and the comparisons made in Fig. 52, we conclude that for an accurate prediction of jet engine inlet acoustic radiation, it is imperative that the physical processes of diffraction and refraction be properly incorporated in the formulation of a theory or a computational model.

\section{Appendix A. Extension of near acoustic field to far field by the surface Green's function method}

For the jet engine inlet noise radiation problem, a good matching surface for the extension of near field to far field is an infinitely long cylindrical surface enclosing the engine. For convenience, we set the matching cylindrical surface to be 3 mesh points inside the computational domain of Fig. 3. The generator of the cylindrical surface is parallel to the line $\mathrm{BD}$. In all the validation directivity computations, the computational domain is extended to a distance of 10 fan diameters in the axial direction from the fan face. This extended domain is 5 diameters larger than the computational domain from the near field pressure contour study. This larger computational domain ensures that most of the sound waves radiated from the engine inlet pass through the matching surface.

Let the diameter of the matching cylindrical surface be $D_{M}$ and the forward flight Mach number be $M_{\infty}$ (i.e., $M_{\infty}=M_{\text {fight }}$ ). Outside the matching cylindrical surface, the linearized Euler equation and energy equations are,

$$
\begin{gathered}
\frac{\partial \boldsymbol{v}}{\partial t}+M_{\infty} \frac{\partial \boldsymbol{v}}{\partial x}=-\nabla p \\
\frac{\partial p}{\partial t}+M_{\infty} \frac{\partial p}{\partial x}+\nabla \bullet \boldsymbol{v}=0
\end{gathered}
$$

Upon eliminating $v$ from Eqs. (A1) and (A2), the governing equation for $p$ is,

$$
\left(\frac{\partial}{\partial t}+M_{\infty} \frac{\partial}{\partial x}\right)^{2} p-\nabla^{2} p=0
$$

The surface Green's function $G\left(r, \phi, x, t ; \phi_{0}, x_{0}, t_{0}\right)$, where $(r, \phi, x, t)$ are the far field observer coordinates and time and $\left(\phi_{0}, x_{0}, t_{0}\right)$ are the source coordinates on the matching surface and time, satisfies the same governing equation as $p$ (Eq. (A3)) together with boundary conditions as follows:

$$
\left(\frac{\partial}{\partial t}+M_{\infty} \frac{\partial}{\partial x}\right)^{2} G-\nabla^{2} G=0
$$

$$
\begin{aligned}
& \text { At }\left(r^{2}+x^{2}\right)^{\frac{1}{\hbar}} \rightarrow \infty, \quad G \text { behaves as outgoing waves } \\
& \text { At } r=\frac{1}{2} D_{M}, \quad G=\frac{\delta\left(x-x_{0}\right) \delta\left(\phi-\phi_{0}\right) \delta\left(t-t_{0}\right)}{\frac{1}{2} D_{M}}
\end{aligned}
$$


Let the radiated pressure field be from a duct mode of azimuthal mode number $m$, radial mode number $n$, and frequency $\Omega$. On the matching surface, the pressure field is,

$$
p=\operatorname{Re}\left\{\hat{p}(x) e^{i(m \phi-\Omega t)}\right\} .
$$

By means of the Green's function, the sound field at a far field point $(r, \phi, x, t)$ is given by,

$$
p(r, \phi, x, t)=\operatorname{Re}\left\{\int_{-\infty}^{\infty} \int_{-\infty}^{\infty} \int_{0}^{2 \pi} \hat{p}\left(x_{0}\right) e^{i\left(m \phi_{0}-\Omega_{0}\right)} G\left(r, \phi, x, t ; \phi_{0}, x_{0}, t_{0}\right) \frac{D_{n}}{2} d \phi_{0} d x_{0} d t_{0}\right\}
$$

To find $G$, let its Fourier transform in $x$ and $t$ be $\hat{G}$, defined as,

$$
\hat{G}\left(r, \phi, k, \omega ; \phi_{0}, x_{0}, t_{0}\right)=\frac{1}{(2 \pi)^{2}} \int_{-\infty}^{\infty} \int_{-\infty}^{\infty} G\left(r, \phi, x, t ; \phi_{0}, x_{0}, t_{0}\right) e^{-i(k x-\omega t)} d x d t
$$

$\hat{G}$ is periodic in $\phi$. Thus, $\hat{G}$ may be expanded as a Fourier series in $\phi$ in the form,

$$
\hat{G}\left(r, \phi, k, \omega ; \phi_{0}, x_{0}, t_{0}\right)=\sum_{n=-\infty}^{\infty} g_{n} e^{i n \phi}
$$

On applying Fourier transforms in $x$ and $t$ to Eq. (A4) and on expanding $\hat{G}$ as in Eq. (A10), it is easy to find that $g_{n}$ is given by the solution of the Bessel equation,

$$
\frac{d^{2} g_{n}}{d r^{2}}+\frac{1}{r} \frac{d g_{n}}{d r}-\frac{n^{2}}{r^{2}} g_{n}+\left[\left(\omega-M_{\infty} k\right)^{2}-k^{2}\right] g_{n}=0
$$

From Eq. (A6), the boundary condition for $g_{n}$ at $r=D_{M} / 2$ is,

$$
g_{n}=\frac{1}{4 \pi^{3} D_{M}} e^{-i\left(k x_{0}+n \phi_{0}-\omega t_{0}\right)}
$$

In deriving Eq. (A12), the $\delta$-function expansion,

$$
\delta\left(\phi-\phi_{0}\right)=\frac{1}{2 \pi} \sum_{n=-\infty}^{\infty} e^{i n\left(\phi-\phi_{0}\right)}
$$

has been used. The solution of Eq. (A11) satisfying radiation boundary condition at $\left(r^{2}+x^{2}\right)^{\frac{1}{2}} \rightarrow \infty$ and boundary condition (A12) is,

$$
g_{n}=\frac{1}{4 \pi^{3} D_{M}} \frac{H_{n}^{(1)}\left[i\left(1-M_{\infty}^{2}\right)^{\frac{1}{2}}\left(k-k_{+}\right)^{\frac{1}{2}}\left(k-k_{-}\right)^{\frac{1}{2}} r\right]}{H^{(1)}\left[\left(1-M_{\infty}^{2}\right)^{\frac{1}{2}}\left(k-k_{+}\right)^{\frac{1}{2}}\left(k-k_{-}\right)^{\frac{1}{2}} \frac{1}{2} D_{M}\right]} e^{-i\left(k k_{0}+n \phi_{0}-\omega t_{0}\right)}
$$

where $k_{+}=\frac{\omega}{1+M_{\infty}}, k_{-}=\frac{-\omega}{1-M_{\infty}}$ and $H_{n}^{(1)}[]$ is the $n^{\text {th }}$-order Hankel function of the first kind. The branch cut of the square root function and the inverse $k$-contour are as shown in Fig. A1. 


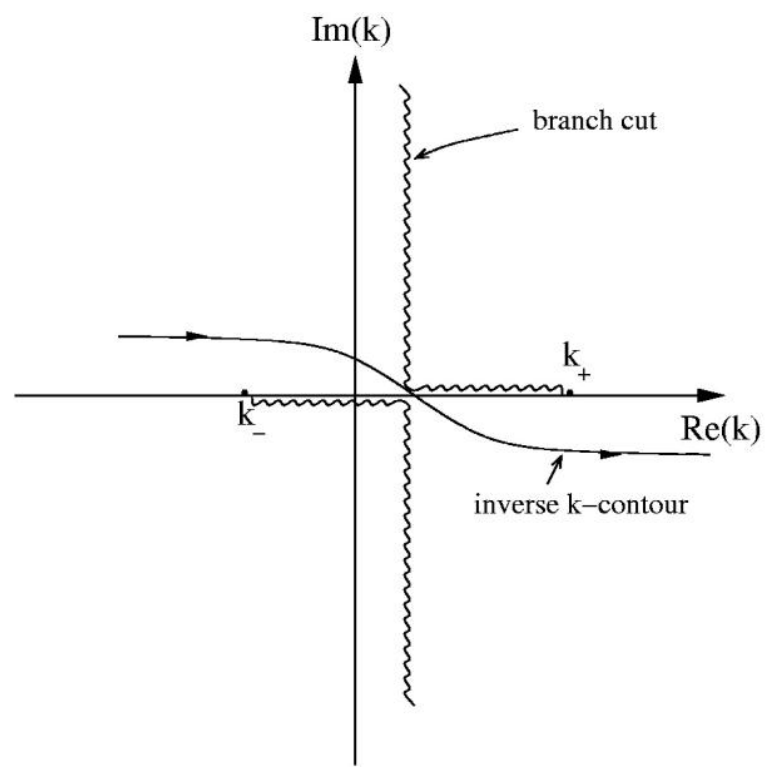

Figure A1. Branch cut for $\left(k-k_{+}\right)^{\frac{1}{2}}\left(k-k_{-}\right)^{\frac{1}{2}}$ in the $k$-plane and the inverse Fourier transform contour.

On substituting solution (A14) into Eq. (A10) and upon performing inverse transforms, the surface Green's function is found to be,

$$
G=\sum_{n=-\infty}^{\infty} \frac{1}{4 \pi^{3} D_{M}} \int_{-\infty}^{\infty} \int_{-\infty}^{\infty} \frac{H_{n}^{(1)}\left[i\left(1-M_{\infty}^{2}\right)^{\frac{1}{2}}\left(k-k_{+}\right)^{\frac{1}{2}}\left(k-k_{-}\right)^{\frac{1}{2}} r\right]}{H_{n}^{(1)}\left[i\left(1-M_{\infty}^{2}\right)^{\frac{1}{2}}\left(k-k_{+}\right)^{\frac{1}{2}}\left(k-k_{-}\right)^{\frac{1}{2}} \frac{1}{2} D_{M}\right]} e^{i\left[k\left(x-x_{0}\right)-\omega\left(t-t_{0}\right)+n\left(\phi-\phi_{0}\right)\right]} d k d \omega
$$

For the far field solution, it is advantageous to switch to spherical polar coordinates $(R, \theta, \phi)$ with the polar axis coinciding with the $x$-axis. The relationship between the spherical polar coordinates and the cylindrical coordinates are,

$$
x=R \cos \theta, \quad r=R \sin \theta
$$

where $\theta$ is the polar angle. Now, for $R \rightarrow \infty, G$, as given by Eq. (A15), may be greatly simplified by first using the asymptotic form of the Hankel function, then evaluating the $k$-integral by the method of stationary phase. The stationary phase point is at $k=\frac{\omega}{1-M_{\infty}^{2}}\left[\frac{\cos \theta}{\left(1-M_{\infty}^{2} \sin ^{2} \theta\right)^{\frac{1}{2}}}-M_{\infty}\right]$. This gives,

$$
\begin{gathered}
G=\frac{1}{2 \pi^{3} D_{M}\left(1-M_{\infty}^{2} \sin ^{2} \theta\right)^{\frac{1}{2}} R} \sum_{n=-\infty}^{\infty} \int_{-\infty}^{\infty} \frac{e^{\left\{\frac{i \omega R}{M_{\infty} \cos \theta+\left(1-M_{\infty}^{2} \sin ^{2} \theta\right)^{\frac{1}{3}}}\right\}} H_{n}^{(1)}\left[\frac{\omega D_{M} \sin \theta}{2\left(1-M_{\infty}^{2} \sin ^{2} \theta\right)^{\frac{1}{2}}}\right]}{e^{\frac{-i \omega}{\left(1-M_{\infty}^{2}\right)}\left[\frac{\cos \theta}{\left(1-M_{\infty}^{2} \sin ^{2} \theta\right)^{\frac{1}{2}}-M_{\infty}}\right] x_{0}}} \\
\times \mathrm{e}^{\mathrm{i}\left[\mathrm{n}\left(\phi-\phi_{0}\right)-\omega\left(t-t_{0}\right)-(n+1) \frac{\pi}{2}\right]} d \omega
\end{gathered}
$$


On inserting the surface Green's function (A17) into Eq. (A8), the far field pressure at $R \rightarrow \infty$ corresponding to a duct mode of azimuthal mode number $m$ and angular frequency $\Omega$ can be found by evaluating the integrals $d \phi_{0} d t_{0} d x_{0} d \omega$. The $d \phi_{0}$ and $d t_{0}$ integration can be carried out easily using,

$$
\begin{gathered}
\int_{0}^{2 \pi} e^{i(m-n) \phi_{0}} d \phi_{0}=2 \pi \delta_{m n} \\
\int_{-\infty}^{\infty} e^{-i(\Omega-\omega) t_{0}} d t_{0}=2 \pi \delta(\omega-\Omega)
\end{gathered}
$$

Because of the delta function $\delta(\omega-\Omega)$ from Eq. (A19), the $d \omega$ integral can also be evaluated. This gives the following formula, which involves a single integral, for the far field pressure,

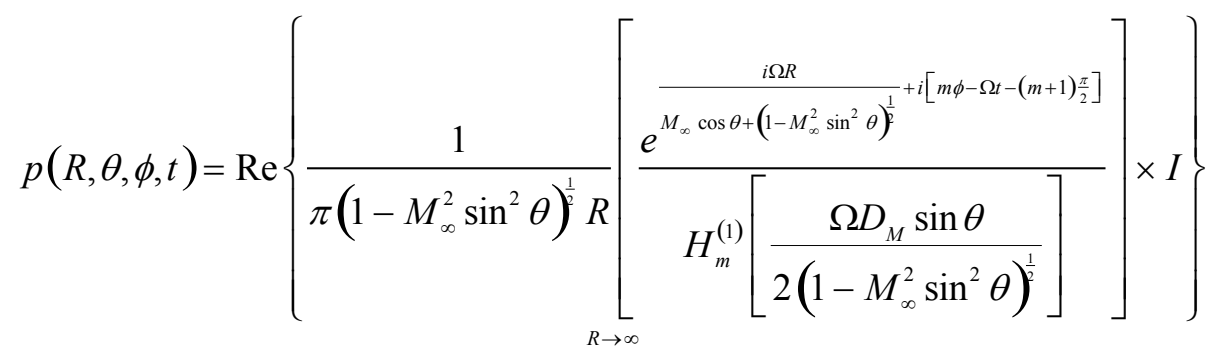

where

$$
I=\int_{-\infty}^{\infty} \hat{p}\left(x_{0}\right) e^{\frac{-i \Omega}{\left(1-M_{\infty}^{2}\right)}\left[\frac{\cos \theta}{\left(1-M_{\infty}^{2} \sin ^{2} \theta\right)^{\frac{1}{2}}-M_{\infty}}\right] x_{0}} d x_{0}
$$

\section{Acknowledgements}

The work done by the third author was funded by the Subsonic Fixed Wing Project of NASA's Fundamental Aeronautics Program. 


\section{References}

${ }^{1}$ Heidmann, M.F., Saule, A.V., and McArdle, J.G., "Predicted and Observed Modal Radiation Pattern from JT15D Engine with Inlet Rods," Journal of Aircraft, Vol. 17, No. 7, 1980, pp. 493-499.

${ }^{2}$ Preisser, J.S., Silcox, R.J., Eversman,W., and Parrett, A.V., "Flight Study of Induced Turbofan Acoustic Radiation with Theoretical Comparisons," Journal of Aircraft, Vol. 22, No. 1, 1985, pp. 57-62.

${ }^{3}$ Herkes, W.H., Olser, R.F., and Uellenberg, S., "The Quite Technology Demonstrator Program: Flight Validation of Airplane Noise-Reduction Concepts," AIAA Paper 2006-2720, May 2006.

${ }^{4}$ Yu, J., Nesbitt, E., Kwan, H.W., Uellenberg, S., Chien, E., Premo, J., Ruiz, M., and Czech, M., "Quite Technology Demonstrator 2 Intake Liner Design and Validation,” AIAA Paper 2006-2458, May 2006.

${ }^{5}$ Callender, B., Janardan, B. Uellenberg, S., Premo, J., Kwan, H.W., Abeysinghe, A., "The Quite Technology Demonstrator Program: Static Test of an Acoustically Smooth Inlet," AIAA Paper 2007-3671, May 2007.

${ }^{6}$ Lan, J., Premo, J. Zlavog, G., Breard, C., Callender, B., and Martinez, M., "Phased Array Measurements of Full-Scale Engine Inlet Noise,” AIAA Paper 2007-3434, May 2007.

${ }^{7}$ Premo, J., and Joppa, P., "Fan Noise Source Diagnostic Test - Wall Measured Circumferential Array Mode Results," AIAA Paper 2002-2429, May 2002.

${ }^{8}$ Heidelberg, L., "Fan Noise Source Diagnostic Test - Tone Modal Structure Results," AIAA Paper 20022428, May 2002.

${ }^{9}$ Woodward, R.P., Huges, C.E., Jeracki, R.J., and Miller, C.J., "Fan Source Diagnostic Test - Far Field Acoustic Results," AIAA Paper 2002-2427, May 2002.

${ }^{10}$ Lansing, D.L., "Exact Solution for Radiation of Sound from a Semi-Infinite Circular Duct with Application to Fan and Compressor Noise," Analytic Methods in Aircraft Aerodynamics, NASA SP-228, 1970, pp. 323-334.

${ }^{11}$ Homicz, G.F. and Lordi, J.A., " A Note on the Radiative Directivity Patterns of Duct Acoustic Modes," Journal of Sound and Vibration, Vol. 41, No. 3, 1975, pp. 283-290.

${ }^{12}$ Kempton, A.J., and Smith, M.G., " Ray Theory Predictions of Sound Radiation form Realistic Engine Intakes," AIAA Paper 81-1982, 1982.

${ }^{13}$ Dougherty , R.P., "Nacelle Acoustic Design by Ray Tracing in Three Dimensions," AIAA Paper 96-1773, May 1996.

${ }^{14}$ Baumeister, K.J., and Horowitz, S.J., "Finite Element-Integral Acoustic Simulation of JT15D Turbofan Engine," ASME Journal of Vibration, Acoustics, Stress, and Reliability in Design, Vol. 106, 1984, pp. 405-413.

${ }^{15}$ Eversman, W., Parret, A.V., Preisser, J.S., and Silcox, R.J., "Contributions to the Finite Element Solution of the Fan Noise Radiation Problem," Transactions of the American Society of Mechanical Engineers, Vol. 107, 1985, pp. 216-223.

${ }^{16}$ Parrett, A., and Eversman, W., "Wave Envelope and Finite Element Approximation for Turbofan Noise Radiation in Flight," AIAA Journal, Vol. 24, No. 5, 1986, pp. 753-760.

${ }^{17}$ Roy, I.D. and Eversman, W., "Improved Finite Element Modeling of the Turbofan Engine Inlet Radiation

Problem," ASME Journal of Vibration and Acoustics, Vol. 117, No. 1. 1995, pp. 109-115.

${ }^{18}$ Ozyoruk, Y., and Long, L.N., " Computation of Sound Radiation from Engine Inlets," AIAA Journal, Vol. 34, No. 5, 1996, pp.894-901.

${ }^{19}$ Ahuja, V., Ozyoruk, Y. and Long, L.N., "Computational Simulations of Fore and Aft Radiation from Ducted Fans," AIAA Paper 2000-1943, May 2000.

${ }^{20}$ Ozyruk, Y., Ahuja, V., and Long, L.N., "Time Domain Simulations of Radiation from Ducted Fans with Liners," AIAA Paper 2001-2171, May 2001.

${ }^{21}$ Zhang, X., Chen, X., Morfey, C.L., and Nelson, P.A., "Computation of Spinning Modal Radiation from an Unflanged Duct," AIAA Paper 2002-2475, May 2002.

${ }^{22}$ Astley, R.J., Hamilton, J.A., Baker, N., and Kitchen, E.H., "Modelling Tone Propagation from Turbofan Inlets - The Effect of Extended Lip Liners," AIAA Paper 2002-2449, May 2002.

${ }^{23}$ Ozyoruk, Y., "Parallel Computation of Forward Radiated Noise of Ducted Fans Including Acoustic Treatment," AIAA Journal, Vol. 40, No. 3, 2002, pp. 450-455.

${ }^{24}$ Ozyoruk, Y., Alpman, E., Ahuja, V. and Long, L.N., "Frequency-Domain Prediction of Turbofan Noise Radiation," Journal of Sound and Vibration, Vol. 270, 2004, 933-950.

${ }^{25}$ Premo, J., Breard, C., and Lan, J., "Prediction of the Inlet Splice Effects from the QDT2 Static Test," AIAA Paper 2007-3544. 
${ }^{26}$ Achunche, I., Astley, J., Sugimoto, R., and Kempton, A., "Prediction of Forward Fan Noise Propagation and Radiation from Intakes," AIAA Paper 2009-3239, May 2009.

${ }^{27}$ Tam, C.K.W. and Ju, H., “Airfoil Tones at Moderate Reynolds Number," Journal of Fluid Mechanics, Vol. 690, 2012, pp. 536-570.

${ }^{28}$ Tam, C.K.W. and Kurbatskii, K.A., "Multi-size-mesh Multi-time-step Dispersion Relation Preserving Scheme for Multiple-scales Aeroacoustics Problems," International Journal of Computational Fluid Dynamics, Vol. 17, 2003, pp. 119-132.

${ }^{29}$ Tam, C.K.W. and Hu, F. Q., "An Optimized Multi-dimensional Interpolation Scheme for Computational Aeroacoustics Applications Using Overset Grid,” AIAA Paper 2004-2812, May 2004.

${ }^{30}$ Tam, C.K.W. and Dong, Z., "Radiation and Outflow Boundary Conditions for Direct Computation of Acoustic and Flow Disturbances in a Nonuniform Mean Flow," Journal of Computational Acoustics, Vol. 4, 1996, 175-201.

${ }^{31}$ Shen, H. and Tam, C.K.W., "Three-dimensional Numerical Simulation of the Jet Screech Phenomenon," AIAA Journal, Vol. 36, No. 1, 2002, pp. 33-41.

${ }^{32}$ Tam, C.K.W., " Advances in Numerical Boundary Conditions for Computational Aeroacoustics,” Journal of Computational Acoustics, Vol. 6, No. 4, 1998, pp. 377-402.

${ }^{33}$ Hu, F.Q., "A Stable Perfectly Matched Layer for Linearized Euler Equations in Unsplit Physical Variables," Journal of Computational Physics, Vol. 173, 2001, 455-480.

${ }^{34}$ Hu F.Q., "Development of PML Absorbing Boundary Conditions for Computational Aeroacoustics: A Progress Review," Computers and Fluids, Vol. 37, 2008, pp. 336-348.

${ }^{35}$ Cantrell, R.H. and Hart, R.W., "Interaction between Sound and Flow in Acoustic Cavities: Mass, Momentum and Energy Considerations," Journal of Acoustic Society of America, Vol. 36, 1964, pp. 697-706.

${ }^{36}$ Heidelberg, L. J., Rice, E. J., Homyak, J., "Acoustic performance of inlet suppressors on an engine generating a single mode," AIAA Paper 81-1965, 1981.

${ }^{37}$ Ffowcs Williams, J. E., and Hawkings, D. L., "Sound Generation by Turbulence and Surfaces in Arbitrary Motion," Proceedings of the Royal Society of London A. Vol. 264, 1969, pp. 321-342.

${ }^{38}$ Pilon, A. R., and Lyrintzis, A. S., "Development of an Improved Kirchhoff Method for Jet Aeroacoustics,“"AIAA Journal. Vol. 36, 1998, pp. 783-790.

${ }^{39}$ Lyrintzis, A. S. "Surface Integral Methods in Computational Aeroacoustics - From the (CFD) Near-field to the (Acoustic) Far-field," International Journal of Aeroacoustics. 2, 2003, 95-128.

${ }^{40}$ Reba, R. A., Narayana, S., Colonius, T., and Suzuki, T., "Modeling Jet Noise from Organized Structures using Near-Field Hydrodynamics Pressure," AIAA Paper 2005-3093, May 2005.

${ }^{41}$ Reba, R. A., Simonich, J., and Schlinker, R., "Measurement of Source Wave-Packets in High-Speed Jets and Connection to Far-Field Sound," AIAA Paper 2008-2891, May 2008.

${ }^{42}$ Tam, C.K.W., Pastouchenko, N.N. and Viswanathan, K., "Continuation of the Near Acoustic Field of a Jet to the Far Field. Part I: Theory," AIAA Paper 2010-3728, May 2010.

${ }^{43}$ Candel, S. M., "Acoustic Radiation from the End of a Two-Dimensional Duct, Effects of Uniform Flow and Duct Lining," Journal of Sound and Vibration, Vol. 28, 1973, pp. 1-13.

${ }^{44}$ Wright, S. E., "Waveguides and Rotating Sources," Journal of Sound and Vibration, Vol. 25, 1972, pp. 163-178.

${ }^{45}$ Rice, E. J., Heidmann, M. F. and Sofrin, T. G., "Modal Propagation Angles in a Cylindrical Duct with Flow and Their Relation to Sound Radiation," AIAA Paper 79-0183, January 1979. 